\title{
LARGE Estimation of the stress-strength reliability of progressively censored inverted exponentiated Rayleigh distributions
}

\author{
A. KOHANSAL
}

\begin{abstract}
Based on progressively Type-II censored samples, this paper deals with the estimation of $R=P(X<Y)$ when $X$ and $Y$ come from two independent inverted exponentiated rayleigh distributions with different shape parameters, but having the same scale parameter. The maximum likelihood estimator and UMVUE of $R$ is obtained. Different confidence intervals are presented. The Bayes estimator of $R$ and the corresponding credible interval using the Gibbs sampling technique are also proposed. Monte Carlo simulations are performed to compare the performances of the different methods. One illustrative example is provided to demonstrate the application of the proposed method.
\end{abstract}

Keywords: Bayesian estimator, Confidence interval, Maximum likelihood estimator, Monte Carlo simulation, Progressive Type-II censoring, Inverted exponentiated rayleigh distribution.

\section{INTRODUCTION}

In reliability analysis, a general problem of interest is inference of the stress-strength parameter $R=P(X<Y)$. The stress $X$ and the strength $Y$ are treated as random variables. The system fails, if at any time the applied stress is greater than its strength. Estimation of the stress-strength parameter has received considerable attention in the statistical literature, starting with the pioneering work of Birnbaum [6]. He provided an interesting connection between the classical Mann-Whitney statistic and the stress-strength model. Since then, work has been accomplished on the estimation and inference of the stress-strength parameter for different distributions from the frequentist and Bayesian points of view. The monograph by Kotz et al. [14] provided a best review of the development of this model till that time. For some of the recent work on the stress-strength model can be obtained in Kundu and Gupta [16; 17], Raqab and Kundu [21], Krishnamoorthy et al. [15], Raqab et al. [22], Kundu and Raqab [18], Lio and Tsai [20].

Type-I and Type-II censoring schemes are the two most popular censoring 
schemes which are used in practice. In Type-I censoring, the test is terminated when a pre-determined time on test has been reached and in Type-II censoring, the test is terminated when a pre-chosen number of failures has been observed. Unfortunately none of these censoring schemes allows the removal of active units during the experiment. Progressive censoring scheme allows the experimenter to remove active units during the experiment. Combining the Type-II censoring and progressive censoring schemes, the progressive Type-II censoring is introduced. Among various censoring schemes, the Type-II progressive censoring scheme has become very popular one in the last decade. It can be described as follows: Suppose $N$ units are placed on a life test and the experimenter decides before hand the quantity $n$, the number of failures to be observed. Now at the time of the first failure, $R_{1}$ of the remaining $N-1$ surviving units are randomly removed from the experiment. At the time of the second failure, $R_{2}$ of the remaining $N-R_{1}-2$ units are randomly removed from the experiment. Finally, at the time of the $n$-th failure, all the remaining surviving units $R_{n}=N-n-R_{1}-\ldots-R_{n-1}$ are removed from the experiment. Therefore, a progressive Type-II censoring scheme consists of $n$, and $\left(R_{1}, \ldots, R_{n}\right)$, such that $R_{1}+\ldots+R_{n}=N-n$. It is clear that this scheme includes the conventional Type-II right censoring scheme (when $R_{1}=\ldots=R_{n-1}=0$ and $R_{n}=N-n$ ) and complete sampling scheme (when $N=n$ and $R_{1}=\ldots=R_{n}=0$ ). For further details on progressively censoring and relevant references, the reader may refer to the book by Balakrishnan and Aggarwala [5].

Based on progressively Type-II censored samples, this paper deals with inference for the stress-strength reliability $R=P(X<Y)$ when $X$ and $Y$ are two independent inverted exponentiated rayleigh distributions with different shape parameters, but having the same scale parameter. For some of the recent work on the stress-strength model based on progressive censored sample can be obtained in Saracoglu et al. [24], Asgharzadeh et al. [3] and Shoaee and Khorram [25]. The inverted exponentiated rayleigh distribution (IERD) with the shape and scale parameters $\alpha$ and $\lambda$, respectively, has the probability density function, cumulative distribution function and failure rate function as:

$$
\begin{array}{llrl}
f(x) & =\frac{2 \alpha \lambda}{x^{3}} e^{-\lambda / x^{2}}\left(1-e^{-\lambda / x^{2}}\right)^{\alpha-1}, & x>0, \alpha, \lambda>0, \\
F(x) & =1-\left(1-e^{-\lambda / x^{2}}\right)^{\alpha}, & x>0, \alpha, \lambda>0, \\
H(x)=\frac{2 \alpha \lambda}{x^{3}\left(e^{\lambda / x^{2}}-1\right)}, & x>0, \alpha, \lambda>0,
\end{array}
$$


respectively. From now on a IERD with the pdf (1) will be denoted by $\operatorname{IER}(\alpha, \lambda)$. The density function and failure rate function of IERD are shown in Figure 1. The failure rate function has a non-monotone unimodal shape. In many practical situations, it is known that the data are coming from a distribution which has a non-monotone failure rate function. Therefore, if the empirical study suggests that the failure rate function of the underlying distribution is non-monotone and it has a unimodal shape, then the IERD may be used to analyse such data sets. In fact, failure rate of the IERD show similar behavior to some well known lifetime models, namely lognormal, inverse Weibull and generalized inverted exponential distribution. So in this respect IERD can be considered as an alternative model to these distributions.
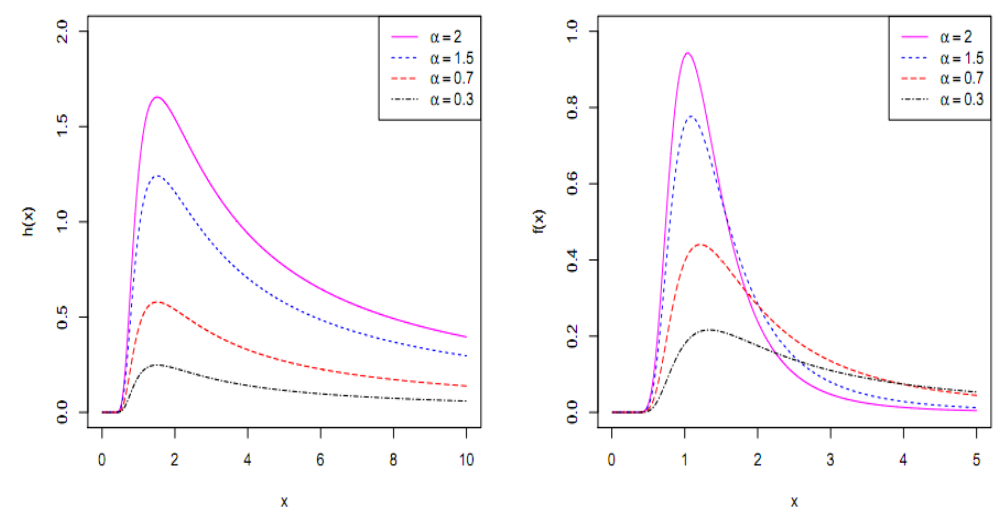

Fig. 1. Shape of density function (right) and failure rate function (left) of IERD when $\lambda=2$

The IERD is a particular member of a general class of inverse exponentiated distribution (see Ghitany et al. [11]). This class includes inverted exponentiated exponential distribution and inverted exponentiated Pareto distribution. Abouammoh and Alshingiti [1] have considered parameter and reliability estimation for inverted exponentiated exponential distribution. They derived many distributional properties and reliability characteristics of inverted exponentiated exponential distribution in a complete sample case. To the best of our knowledge, the class of inverse exponentiated distribution has not received much attention for censored data. Recently, Rastogi and Tripathi [23] considered the estimation for an IERD under type II progressive censoring. We study the inference of the stress-strength parameter $R=P(X<Y)$ when $X$ and $Y$ are independent inverted exponentiated rayleigh 
random variables. It is further assumed that we observe progressively Type-II censored samples from both distributions. We can formulate the problem as follows: It is assumed that $X$ and $Y$ are independent inverted exponentiated rayleigh random variables with common scale parameter $\lambda$ and shape parameters $\alpha$ and $\beta$, respectively. There are two progressive censoring schemes, $\left\{N, n, R_{1}, R_{2}, \ldots, R_{n}\right\}$ and $\left\{M, m, S_{1}, S_{2}, \ldots, S_{m}\right\}$ for $X$ and $Y$, respectively. We observe progressively censored sample $\left\{X_{1: n: N}, \ldots, X_{n: n: N}\right\}$ from $X$ and $\left\{Y_{1: m: M}, \ldots, Y_{m: m: M}\right\}$ from $Y$ based on the above progressive censored samples, our problem is to estimate $R=P(X<Y)=\frac{\alpha}{\alpha+\beta}$ (see, e.g. [24], [25]).

The layout of this paper is as follows: In Section 2, we discuss the MLE of $R$. It is observed that the MLE can be achieved by solving a non-linear equation and it can be obtained using an iterative procedure. The uniformly minimum variance unbiased estimator (UMVUE) of $R$ is derived in Section 3. The exact, asymptotic and two bootstrap confidence intervals of $R$ are presented in Section 4. Bayes estimate and the associated credible interval are discussed in Section 5. Simulation results and data analysis are presented in Sections 6. Finally, we conclude the paper in Section 7.

\section{MAXIMUM LIKELIHOOD ESTIMATION OF $R$}

Let $X \sim \operatorname{IER}(\alpha, \lambda)$ and $Y \sim \operatorname{IER}(\beta, \lambda)$ be independent random variables. Then it can be easily seen that the reliability function is $R=P(X<Y)=\frac{\alpha}{\alpha+\beta}$. Our interest is in estimating $R$ based on progressive Type-II censored data on both variables.

To derive the MLE of $R$, first we obtain the MLEs of $\alpha, \beta$ and $\lambda$. Let $\left\{X_{1: n: N}, \ldots, X_{n: n: N}\right\}$ be a progressively censored sample from $\operatorname{IER}(\alpha, \lambda)$ under the progressive censoring scheme

$\left\{N, n, R_{1}, R_{2}, \ldots, R_{n}\right\}$. Similarly, let $\left\{Y_{1: m: M}, \ldots, Y_{m: m: M}\right\}$ be a progressively censored sample from $\operatorname{IER}(\beta, \lambda)$ under the progressive censoring scheme $\left\{M, m, S_{1}, S_{2}, \ldots, S_{m}\right\}$. Therefore, from [24] the likelihood function of $\alpha, \beta$ and $\lambda$ is given by

$$
L(\alpha, \beta, \lambda)=\left[c_{1} \prod_{i=1}^{n} f\left(x_{i}\right)\left[1-F\left(x_{i}\right)\right]^{R_{i}}\right] \times\left[c_{2} \prod_{j=1}^{m} f\left(y_{j}\right)\left[1-F\left(y_{j}\right)\right]^{S_{j}}\right],
$$

where

$$
\begin{aligned}
& c_{1}=N\left(N-R_{1}-1\right) \cdots\left(N-R_{1}-\cdots-R_{n-1}-n+1\right), \\
& c_{2}=M\left(M-S_{1}-1\right) \cdots\left(M-S_{1}-\cdots-S_{m-1}-m+1\right) .
\end{aligned}
$$


The likelihood function of the observed data as follows:

$$
\begin{aligned}
L(\text { data } \mid \alpha, \beta, \lambda)= & c_{1} c_{2} 2^{n+m} \alpha^{n} \beta^{m} \lambda^{n+m}\left(\prod_{i=1}^{n} \frac{1}{x_{i}^{3}}\right)\left(\prod_{j=1}^{m} \frac{1}{y_{j}^{3}}\right) \exp \left\{-\lambda\left(\sum_{i=1}^{n} \frac{1}{x_{i}^{2}}+\sum_{j=1}^{m} \frac{1}{y_{j}^{2}}\right)\right\} \\
& \times\left(\prod_{i=1}^{n}\left(1-e^{-\lambda / x_{i}^{2}}\right)^{\alpha\left(R_{i}+1\right)-1}\right)\left(\prod_{j=1}^{m}\left(1-e^{-\lambda / y_{j}^{2}}\right)^{\beta\left(S_{j}+1\right)-1}\right) .
\end{aligned}
$$

From (2), the log-likelihood function is

$$
\begin{aligned}
\ell(\alpha, \beta, \lambda)= & \text { Constant }+n \log (\alpha)+m \log (\beta)+(n+m) \log (\lambda)-\lambda\left(\sum_{i=1}^{n} \frac{1}{x_{i}^{2}}+\sum_{j=1}^{m} \frac{1}{y_{j}^{2}}\right) \\
& -3\left(\sum_{i=1}^{n} \log \left(x_{i}\right)+\sum_{j=1}^{m} \log \left(y_{j}\right)\right)+\sum_{i=1}^{n}\left(\alpha\left(R_{i}+1\right)-1\right) \log \left(1-e^{-\lambda / x_{i}^{2}}\right) \\
& +\sum_{j=1}^{m}\left(\beta\left(S_{j}+1\right)-1\right) \log \left(1-e^{-\lambda / y_{j}^{2}}\right) .
\end{aligned}
$$

So, the MLEs of $\alpha, \beta$ and $\lambda$, say $\widehat{\alpha}, \widehat{\beta}$ and $\widehat{\lambda}$, respectively, can be obtained as the solution of

$$
\begin{aligned}
\frac{\partial \ell}{\partial \alpha}= & \frac{n}{\alpha}+\sum_{i=1}^{n}\left(R_{i}+1\right) \log \left(1-e^{-\lambda / x_{i}^{2}}\right)=0, \\
\frac{\partial \ell}{\partial \beta}= & \frac{m}{\beta}+\sum_{j=1}^{m}\left(S_{j}+1\right) \log \left(1-e^{-\lambda / y_{j}^{2}}\right)=0, \\
\frac{\partial \ell}{\partial \lambda}= & \frac{n+m}{\lambda}-\left(\sum_{i=1}^{n} \frac{1}{x_{i}^{2}}+\sum_{j=1}^{m} \frac{1}{y_{j}^{2}}\right)+\sum_{i=1}^{n} \frac{\left(\alpha\left(R_{i}+1\right)-1\right) e^{-\lambda / x_{i}^{2}}}{x_{i}^{2}\left(1-e^{-\lambda / x_{i}^{2}}\right)} \\
& +\sum_{j=1}^{m} \frac{\left(\beta\left(S_{j}+1\right)-1\right) e^{-\lambda / y_{j}^{2}}}{y_{j}^{2}\left(1-e^{-\lambda / y_{j}^{2}}\right)} .
\end{aligned}
$$

From (3) and (4), we obtain

$$
\widehat{\alpha}(\lambda)=-\frac{n}{\sum_{i=1}^{n}\left(R_{i}+1\right) \log \left(1-e^{-\lambda / x_{i}^{2}}\right)}, \quad \widehat{\beta}(\lambda)=-\frac{m}{\sum_{j=1}^{m}\left(S_{j}+1\right) \log \left(1-e^{-\lambda / y_{j}^{2}}\right)},
$$


and $\widehat{\lambda}$ can be found as the solution of the non-linear equation $k(\lambda)=\lambda$, where

$$
\begin{array}{r}
k(\lambda)=(n+m)\left\{\sum_{i=1}^{n} \frac{1}{x_{i}^{2}}\left(1-\frac{\left(\alpha\left(R_{i}+1\right)-1\right) e^{-\lambda / x_{i}^{2}}}{1-e^{-\lambda / x_{i}^{2}}}\right)\right. \\
\left.+\sum_{j=1}^{m} \frac{1}{y_{j}^{2}}\left(1-\frac{\left(\beta\left(S_{j}+1\right)-1\right) e^{-\lambda / y_{j}^{2}}}{1-e^{-\lambda / y_{j}^{2}}}\right)\right\}^{-1} .
\end{array}
$$

Since, $\widehat{\lambda}$ is a fixed point solution of the above non-linear equation, therefore, it can be resulted using an iterative scheme as $k\left(\lambda_{(j)}\right)=\lambda_{(j+1)}$, where $\lambda_{(j)}$ is the $j$-th iterate of $\widehat{\lambda}$. The iteration procedure should be stopped when $\left|\lambda_{(j)}-\lambda_{(j+1)}\right|$ becomes sufficiently small. Once we obtain $\widehat{\lambda}$, then $\widehat{\alpha}$ and $\widehat{\beta}$ can be resulted. Therefore, the MLE of $R$ becomes

$$
\widehat{R}=\frac{\widehat{\alpha}}{\widehat{\alpha}+\widehat{\beta}}
$$

\section{UMVUE OF $R$}

In this section, the UMVUE of $R$ is derived. Let $\left\{X_{1: n: N}, \ldots, X_{n: n: N}\right\}$ be a progressively censored sample from $\operatorname{IER}(\alpha, \lambda)$ under the progressive censoring scheme $\left\{N, n, R_{1}, \ldots, R_{n}\right\}$. Similarly, let $\left\{Y_{1: m: M}, \ldots, Y_{m: m: M}\right\}$ be a progressively censored sample from $\operatorname{IER}(\beta, \lambda)$ under the progressive censoring scheme $\left\{M, m, S_{1}, \ldots, S_{m}\right\}$. The joint pdf of $X_{1: n: N}, \ldots, X_{n: n: N}$ is

$$
\begin{aligned}
f_{X_{1: n: N}, \ldots, X_{n: n: N}}\left(x_{1}, \ldots, x_{n}\right) & =c_{1}(2 \alpha \lambda)^{n}\left(\prod_{i=1}^{n} \frac{1}{x_{i}^{3}}\right)\left(\prod_{i=1}^{n} \frac{1}{1-e^{-\lambda / x_{i}^{2}}}\right) \exp \left\{-\lambda \sum_{i=1}^{n} \frac{1}{x_{i}^{2}}\right\} \\
& \times \exp \left\{\sum_{i=1}^{n} \alpha\left(R_{i}+1\right) \log \left(1-e^{-\lambda / x_{i}^{2}}\right)\right\},
\end{aligned}
$$

where $0<x_{1}<\ldots<x_{n}<\infty$ (see [5], for more details). It is immediate from (7) that $U=-\sum_{i=1}^{n}\left(R_{i}+1\right) \log \left(1-e^{-\lambda / x_{i}^{2}}\right)$ is the complete sufficient statistics for $\alpha$ when $\lambda$ is known. It is easy to see that

$$
X_{i: n: N}^{*}=-\log \left(1-e^{-\lambda / X_{i: n: N}^{2}}\right), \quad i=1, \ldots, n,
$$


be a progressive censored sample from an exponential distribution with mean $\alpha^{-1}$. Let

$$
\begin{aligned}
Z_{1} & =N X_{1: n: N}^{*}, \\
Z_{2} & =\left(N-R_{1}-1\right)\left(X_{2: n: N}^{*}-X_{1: n: N}^{*}\right), \\
& \vdots \\
Z_{n} & =\left(N-R_{1}-\ldots-R_{n-1}-n+1\right)\left(X_{n: n: N}^{*}-X_{n-1: n: N}^{*}\right) .
\end{aligned}
$$

From Cao and Cheng [7], we get that $Z_{1}, \ldots, Z_{n}$ are independent and identically distributed exponential random variables with mean $\alpha^{-1}$. Moreover $U=\sum_{i=1}^{n} Z_{i}=\sum_{i=1}^{n}\left(R_{i}+1\right) X_{i: n: N}^{*}$ has a gamma distribution with the shape parameter $n$ and the scale parameter $\alpha$, in symbols $U \sim \Gamma(n, \alpha)$, i.e. it has the pdf

$$
f_{U}(u)=\frac{\alpha^{n}}{\Gamma(n)} u^{n-1} e^{-\alpha u}, u>0 .
$$

LEMMA 1. Let $Y_{j: m: M}^{*}=-\log \left(1-e^{-\lambda / Y_{j: m: M}^{2}}\right)$ and $V=\sum_{j=1}^{m}\left(S_{j}+1\right) Y_{j: m: M}^{*}$. The conditional pdf of $X_{1: n: N}^{*}$ given $U=u$, is

$$
f_{X_{1: n: N}^{*} \mid U=u}(x)=N(n-1) \frac{(u-N x)^{n-2}}{u^{n-1}}, 0<x<u / N,
$$

and the conditional pdf of $Y_{1: m: M}^{*}$ given $V=v$, is

$$
f_{Y_{1: m: M}^{*} \mid V=v}(y)=M(m-1) \frac{(v-M y)^{m-2}}{v^{m-1}}, 0<y<v / M .
$$

PROOF. We will prove the first part, second part follows along the same line. Note that

$$
f_{X_{1: n: N}^{*} \mid U=u}(x)=\frac{f_{X_{1: n: N}^{*}, U}(x, u)}{f_{U}(u)},
$$

where $f_{X_{1: n: N}^{*}, U}(x, u)$ is the the joint pdf of $X_{1: n: N}^{*}$ and $U$ and $f_{U}(u)$ is the pdf of $U$. It is obvious that $U$ is a complete sufficient statistics for $\alpha$ when $\lambda$ is known. Suppose we denote $W=\sum_{i=2}^{n} Z_{i}$ then clearly $W$ and $Z_{1}$ are independent. The joint pdf of $X_{1: n: N}^{*}$ and $U$ can be easily obtained from the joint pdf of $W$ and $Z_{1}$, by using the the transformation $Z_{1}=N X_{1: n: N}^{*}$ and $U=W+Z_{1}$. Finally the result is found using (8).

THEOREM 1. Based on the complete sufficient statistics $U$ and $V$, as defined before for $\alpha$ and $\beta$ respectively, the UMVUE of $R$, say $\tilde{R}$, for $n \geq 2$, and $m \geq 2$, can 
be expressed as follows:

$$
\tilde{R}=\left\{\begin{array}{r}
1-\sum_{k=0}^{n-1}(-1)^{k}\left(\frac{v}{u}\right)^{k} \frac{\left(\begin{array}{c}
n-1 \\
k
\end{array}\right)}{\left(\begin{array}{c}
m+k-1 \\
k
\end{array}\right)} \text { if } v<u, \\
\sum_{k=0}^{m-1}(-1)^{k}\left(\frac{u}{v}\right)^{k} \frac{\left(\begin{array}{c}
m-1 \\
k
\end{array}\right)}{\left(\begin{array}{c}
n+k-1 \\
k
\end{array}\right)} \text { if } v>u .
\end{array}\right.
$$

PROOF. Observe that $X_{1: n: N}^{*}$ and $Y_{1: m: M}^{*}$ follow an exponential distribution with mean $(N \alpha)^{-1}$ and $(M \beta)^{-1}$, respectively, therefore,

$$
\phi\left(X_{1}^{*}, Y_{1}^{*}\right)=\left\{\begin{array}{l}
1 \text { if } M Y_{1: m: M}^{*}>N X_{1: n: N}^{*}, \\
0 \text { if } M Y_{1: m: M}^{*}<N X_{1: n: N}^{*},
\end{array}\right.
$$

is an unbiased estimator of $R$. Therefore,

$$
\tilde{R}=E\left[\phi\left(X_{1}^{*}, Y_{1}^{*}\right) \mid U=u, V=v\right]=\iint_{\mathscr{A}} f_{X_{1}^{*} \mid U=u}(x) f_{Y_{1}^{*} \mid V=v}(y) d x d y,
$$

where $\mathscr{A}=\{(x, y): 0<x<u / N, 0<y<v / M, N x<M y\}, f_{X_{1}^{*} \mid U=u}(x)$ and $f_{Y_{1}^{*} \mid V=v}(y)$ are same as defined in Lemma 1. For $v<u$

$$
\begin{aligned}
\tilde{R} & =\frac{N(n-1)}{u^{n-1}} \frac{M(m-1)}{v^{m-1}} \int_{0}^{v / M} \int_{0}^{M y / N}(u-N x)^{n-2}(v-M y)^{m-2} d x d y \\
& =1-\frac{M(m-1)}{u^{n-1} v^{m-1}} \int_{0}^{v / M}(v-M y)^{m-2}(u-M y)^{n-1} d y \quad\left\{P u t: \frac{M y}{v}=t\right\} \\
& =1-(m-1) \int_{0}^{1}(1-t)^{m-2}\left(1-\frac{v t}{u}\right)^{n-1} d t \\
& =1-(m-1) \int_{0}^{1}(1-t)^{m-2} \sum_{k=0}^{n-1}(-1)^{k}\left(\begin{array}{c}
n-1 \\
k
\end{array}\right)\left(\frac{v t}{u}\right)^{k} d t \\
& =1-\sum_{k=0}^{n-1}(-1)^{k}\left(\frac{v}{u}\right)^{k} \frac{\left(\begin{array}{c}
n-1 \\
k
\end{array}\right)}{\left(\begin{array}{c}
m+k-1 \\
k
\end{array}\right)} .
\end{aligned}
$$

Similarly for $v>u, \tilde{R}=\sum_{k=0}^{m-1}(-1)^{k}\left(\frac{u}{v}\right)^{k} \frac{\left(\begin{array}{c}m-1 \\ k\end{array}\right)}{\left(\begin{array}{c}n+k-1 \\ k\end{array}\right)}$. 


\section{CONFIDENCE INTERVALS}

\subsection{Exact confidence interval}

Let $\left\{X_{1: n: N}, \ldots, X_{n: n: N}\right\}$ be a progressively censored sample from $\operatorname{IER}(\alpha, \lambda)$ under the progressive censoring scheme $\left\{N, n, R_{1}, \ldots, R_{n}\right\}$. Similarly, let $\left\{Y_{1: m: M}, \ldots, Y_{m: m: M}\right\}$ be a progressively censored sample from $\operatorname{IER}(\beta, \lambda)$ under the progressive censoring scheme $\left\{M, m, S_{1}, \ldots, S_{m}\right\}$. Let $X_{i: n: N}^{* *}=-\alpha \log \left(1-e^{-\lambda / X_{i: n: N}^{2}}\right)$, $i=1, \cdots, n\left(\right.$ or $\left.Y_{j: m: M}^{* *}=-\beta \log \left(1-e^{-\lambda / Y_{j: m: M}^{2}}\right), j=1, \cdots, m\right)$. It is easy to see that $X_{i: n: N}^{* *}, i=1, \cdots, n$ (or $Y_{j: m: M}^{* *}, j=1, \cdots, m$ ) is a progressive censoring sample from a standard exponential distribution. Let us consider the following transformations:

$$
\begin{aligned}
& Z_{1}^{*}=N X_{1: n: N}^{* *}, Z_{i}^{*}=N-\sum_{k=1}^{i-1}\left(R_{k}+1\right)\left(X_{i: n: N}^{* *}-X_{i-1: n: N}^{* *}\right), i=2, \cdots, n, \\
& D_{1}^{*}=M Y_{1: m: M}^{* *}, D_{j}^{*}=M-\sum_{k=1}^{j-1}\left(S_{k}+1\right)\left(Y_{j: m: M}^{* *}-Y_{j-1: m: M}^{* *}\right), j=2, \cdots, m .
\end{aligned}
$$

From Cao and Cheng [7], we get that $Z_{1}^{*}, \cdots, Z_{n}^{*}\left(\right.$ or $D_{1}^{*}, \cdots, D_{m}^{*}$ ) are independent and identically distribution as standard exponential.

Let $V_{X}=2 Z_{1}^{*}$ and $U_{X}=2 \sum_{i=2}^{n} Z_{i}^{*}\left(\right.$ or $V_{Y}=2 D_{1}^{*}$ and $U_{Y}=2 \sum_{j=2}^{m} D_{j}^{*}$ ). Then $V_{X}$ and $U_{X}$ (or $V_{Y}$ and $U_{Y}$ ) are independent random variables and

$$
V_{X} \sim \chi_{(2)}^{2} \text { and } U_{X} \sim \chi_{(2 n-2)}^{2}\left(\text { or } V_{Y} \sim \chi_{(2)}^{2} \text { and } U_{Y} \sim \chi_{(2 m-2)}^{2}\right) .
$$

LEMMA 2. Let $T_{X}(\lambda)=\frac{U_{X}}{(n-1) V_{X}}, T_{1}=U_{X}+V_{X}, T_{Y}(\lambda)=\frac{U_{Y}}{(m-1) V_{Y}}$ and $T_{2}=U_{Y}+$ $V_{Y}$. We can find that

$$
T_{X}(\lambda) \sim F(2 n-2,2), T_{1} \sim \chi^{2}(2 n), T_{Y}(\lambda) \sim F(2 m-2,2) \text { and } T_{2} \sim \chi^{2}(2 m) .
$$

It is obvious that $T_{X}(\lambda)$ and $T_{Y}(\lambda)$ are independent. Furthermore, Johnson et al. [13] indicated that $T_{X}(\lambda)$ and $T_{1}\left(\right.$ or $T_{Y}(\lambda)$ and $\left.T_{2}\right)$ are independent.

LEMMA 3. $T_{X}(\lambda)\left(\right.$ or $\left.T_{Y}(\lambda)\right)$ is strictly decreasing in $\lambda$.

PROOF. Let $\xi(\lambda)=\frac{\log \left(1-e^{-\lambda / a_{i}^{2}}\right)}{\log \left(1-e^{-\lambda / a_{1}^{2}}\right)}: 0<a_{1}<a_{i}, i=2, \ldots, n$. This function is 
strictly decreasing in $\lambda$ since the first derivative of $\xi(\lambda)$ is

$$
\begin{aligned}
\frac{d \xi(\lambda)}{d \lambda} & =\frac{1}{\log ^{2}\left(1-e^{-\lambda / a_{1}^{2}}\right)}\left[\log \left(1-e^{-\lambda / a_{1}^{2}}\right) \frac{e^{-\lambda / a_{i}^{2}}}{a_{i}^{2}\left(1-e^{-\lambda / a_{i}^{2}}\right)}-\log \left(1-e^{-\lambda / a_{i}^{2}}\right) \frac{e^{-\lambda / a_{1}^{2}}}{a_{1}^{2}\left(1-e^{-\lambda / a_{1}^{2}}\right)}\right] \\
& =\frac{-1}{\log ^{2}\left(1-e^{-\lambda / a_{1}^{2}}\right)}\left[\log \left(\frac{1}{1-e^{-\lambda / a_{1}^{2}}}\right) \frac{e^{-\lambda / a_{i}^{2}}}{a_{i}^{2}\left(1-e^{-\lambda / a_{i}^{2}}\right)}-\log \left(\frac{1}{1-e^{-\lambda / a_{i}^{2}}}\right) \frac{e^{-\lambda / a_{1}^{2}}}{a_{1}^{2}\left(1-e^{-\lambda / a_{1}^{2}}\right)}\right] \\
& <\frac{-1}{\log ^{2}\left(1-e^{-\lambda / a_{1}^{2}}\right)}\left[\log \left(\frac{1}{1-e^{-\lambda / a_{i}^{2}}}\right) \frac{e^{-\lambda / a_{i}^{2}}}{a_{1}^{2}\left(1-e^{-\lambda / a_{i}^{2}}\right)}-\log \left(\frac{1}{1-e^{-\lambda / a_{i}^{2}}}\right) \frac{e^{-\lambda / a_{1}^{2}}}{a_{1}^{2}\left(1-e^{-\lambda / a_{1}^{2}}\right)}\right] \\
& =\frac{-1}{a_{1}^{2} \log ^{2}\left(1-e^{-\lambda / a_{1}^{2}}\right)} \cdot \log \left(\frac{1}{1-e^{-\lambda / a_{i}^{2}}}\right)\left[\frac{e^{-\lambda / a_{i}^{2}}}{1-e^{-\lambda / a_{i}^{2}}}-\frac{e^{-\lambda / a_{1}^{2}}}{1-e^{-\lambda / a_{1}^{2}}}\right]<0 .
\end{aligned}
$$

Moreover, after simplifying $T_{X}(\lambda)$, this expression becomes

$$
\begin{aligned}
T_{X}(\lambda) & =\frac{U_{X}}{(n-1) V_{X}}=\frac{\sum_{i=1}^{n} Z_{i}^{*}-Z_{1}^{*}}{(n-1) Z_{1}^{*}} \\
& =\frac{1}{N(n-1)} \sum_{i=1}^{n}\left(R_{i}+1\right) \frac{\log \left(1-e^{-\lambda / X_{i: n: N}^{2}}\right)}{\log \left(1-e^{-\lambda / X_{1: n: N}^{2}}\right)}-\frac{1}{n-1} .
\end{aligned}
$$

Hence, it is easy to see that $T_{X}(\lambda)$ is a strictly decreasing function of $\lambda$.

LEMMA 4. When $\lambda$ is known, the MLE of R can be obtained as

$$
\widehat{R}_{M L}=\frac{1}{1+\frac{m T_{1} / \alpha}{n T_{2} / \beta}} .
$$

It is obvious that $T_{1}$ and $T_{2}$ are independent. Alternatively, $T_{X}(\lambda), T_{Y}(\lambda)$ and $\widehat{R}_{M L}$ are independent. Also, by using Lemma 2, we have

$$
\widehat{R}_{M L}=\frac{1}{1+\frac{\beta}{\alpha} F}, \text { or } F=\frac{R}{1-R} \cdot \frac{1-\widehat{R}}{\widehat{R}},
$$

where $F$ has Fisher distribution with $(2 n, 2 m)$ degrees of freedom, in symbols $F \sim$ $F(2 n, 2 m)$.

THEOREM 2. Suppose that $\left\{X_{1: n: N}, \ldots, X_{n: n: N}\right\}$ be a progressively censored sample from $\operatorname{IER}(\alpha, \lambda)$ and $\left\{Y_{1: m: M}, \ldots, Y_{m: m: M}\right\}$ be a progressively censored sample from $\operatorname{IER}(\beta, \lambda)$. Then 
(i). for any $0<\gamma<1$,

$$
\begin{aligned}
\left(\operatorname { m a x } \left\{T_{X}^{-1}\right.\right. & \left.\left(F_{(1+\sqrt{1-\gamma}) / 2}(2 n-2,2)\right), T_{Y}^{-1}\left(F_{(1+\sqrt{1-\gamma}) / 2}(2 m-2,2)\right)\right\} \\
& \left.\min \left\{T_{X}^{-1}\left(F_{(1-\sqrt{1-\gamma}) / 2}(2 n-2,2)\right), T_{Y}^{-1}\left(F_{(1-\sqrt{1-\gamma}) / 2}(2 m-2,2)\right)\right\}\right)
\end{aligned}
$$

is a $100(1-\gamma) \%$ confidence interval for $\lambda$, where $F_{\gamma}(p, q)$ is $100 \gamma$-th percentile of $F(p, q)$.

(ii). for any $0<\gamma<1$, the following inequalities determine a $100(1-\gamma) \%$ joint confidence region for $(\lambda, R)$,

$$
\left\{\begin{array}{c}
\max \left\{T_{X}^{-1}\left(F_{(1+\sqrt[4]{1-\gamma}) / 2}(2 n-2,2)\right), T_{Y}^{-1}\left(F_{(1+\sqrt[4]{1-\gamma}) / 2}(2 m-2,2)\right)\right\} \leq \lambda \\
\leq \min \left\{T_{X}^{-1}\left(F_{(1-\sqrt[4]{1-\gamma}) / 2}(2 n-2,2)\right), T_{Y}^{-1}\left(F_{(1-\sqrt[4]{1-\gamma}) / 2}(2 m-2,2)\right)\right\} \\
\frac{1}{1+\frac{1-\widehat{R}_{M L}}{\widehat{R}_{M L}} F_{1-(1-\sqrt{1-\gamma}) / 2}(2 m, 2 n)} \leq R \leq \frac{1}{1+\frac{1-\widehat{R}_{M L}}{\widehat{R}_{M L}} F_{1-(1+\sqrt{1-\gamma}) / 2}(2 m, 2 n)}
\end{array}\right.
$$

PROOF.

(i). By using Lemma 2 and Lemma 3, we have

$$
\begin{aligned}
& 1-\gamma= \sqrt{1-\gamma} \cdot \sqrt{1-\gamma} \\
&= P\left[F_{(1-\sqrt{1-\gamma}) / 2}(2 n-2,2) \leq T_{X}(\lambda) \leq F_{(1+\sqrt{1-\gamma}) / 2}(2 n-2,2)\right] \\
& \quad \times P\left[F_{(1-\sqrt{1-\gamma}) / 2}(2 m-2,2) \leq T_{Y}(\lambda) \leq F_{(1+\sqrt{1-\gamma}) / 2}(2 m-2,2)\right] \\
&= P\left[F_{(1-\sqrt{1-\gamma}) / 2}(2 n-2,2) \leq T_{X}(\lambda) \leq F_{(1+\sqrt{1-\gamma}) / 2}(2 n-2,2),\right. \\
&\left.\quad F_{(1-\sqrt{1-\gamma}) / 2}(2 m-2,2) \leq T_{Y}(\lambda) \leq F_{(1+\sqrt{1-\gamma}) / 2}(2 m-2,2)\right] \\
&= P\left[T_{X}^{-1}\left(F_{(1+\sqrt{1-\gamma}) / 2}(2 n-2,2)\right) \leq \lambda \leq T_{X}^{-1}\left(F_{(1-\sqrt{1-\gamma}) / 2}(2 n-2,2)\right),\right. \\
&\left.\quad T_{Y}^{-1}\left(F_{(1+\sqrt{1-\gamma}) / 2}(2 m-2,2)\right) \leq \lambda \leq T_{Y}^{-1}\left(F_{(1-\sqrt{1-\gamma}) / 2}(2 m-2,2)\right)\right] \\
&= P\left[\max \left\{T_{X}^{-1}\left(F_{(1+\sqrt{1-\gamma}) / 2}(2 n-2,2)\right), T_{Y}^{-1}\left(F_{(1+\sqrt{1-\gamma}) / 2}(2 m-2,2)\right)\right\} \leq \lambda\right. \\
&\left.\quad \leq \min \left\{T_{X}^{-1}\left(F_{(1-\sqrt{1-\gamma}) / 2}(2 n-2,2)\right), T_{Y}^{-1}\left(F_{(1-\sqrt{1-\gamma}) / 2}(2 m-2,2)\right)\right\}\right] .
\end{aligned}
$$


(ii). By using Lemma 2, Lemma 3 and Lemma 4, we have

$$
\begin{aligned}
& 1-\gamma=\sqrt[4]{1-\gamma} \cdot \sqrt[4]{1-\gamma} \cdot \sqrt{1-\gamma} \\
& =P\left[F_{(1-\sqrt[4]{1-\gamma}) / 2}(2 n-2,2) \leq T_{X}(\lambda) \leq F_{(1+\sqrt[4]{1-\gamma}) / 2}(2 n-2,2)\right] \\
& \times P\left[F_{(1-\sqrt[4]{1-\gamma}) / 2}(2 m-2,2) \leq T_{Y}(\lambda) \leq F_{(1+\sqrt[4]{1-\gamma}) / 2}(2 m-2,2)\right] \\
& \times P\left[F_{(1-\sqrt{1-\gamma}) / 2}(2 n, 2 m) \leq F \leq F_{(1+\sqrt{1-\gamma}) / 2}(2 n, 2 m)\right] \\
& =P\left[F_{(1-\sqrt[4]{1-\gamma}) / 2}(2 n-2,2) \leq T_{X}(\lambda) \leq F_{(1+\sqrt[4]{1-\gamma}) / 2}(2 n-2,2),\right. \\
& F_{(1-\sqrt[4]{1-\gamma}) / 2}(2 m-2,2) \leq T_{Y}(\lambda) \leq F_{(1+\sqrt[4]{1-\gamma}) / 2}(2 m-2,2) \\
& \left.F_{(1-\sqrt{1-\gamma}) / 2}(2 n, 2 m) \leq \frac{R}{1-R} \cdot \frac{1-\widehat{R}_{M L}}{\widehat{R}_{M L}} \leq F_{(1+\sqrt{1-\gamma}) / 2}(2 n, 2 m)\right] \\
& =P\left[T_{X}^{-1}\left(F_{(1+\sqrt[4]{1-\gamma}) / 2}(2 n-2,2)\right) \leq \lambda \leq T_{X}^{-1}\left(F_{(1-\sqrt[4]{1-\gamma}) / 2}(2 n-2,2)\right),\right. \\
& T_{Y}^{-1}\left(F_{(1+\sqrt[4]{1-\gamma}) / 2}(2 m-2,2)\right) \leq \lambda \leq T_{Y}^{-1}\left(F_{(1-\sqrt[4]{1-\gamma}) / 2}(2 m-2,2)\right), \\
& \left.F_{1-(1+\sqrt{1-\gamma}) / 2}(2 m, 2 n) \leq \frac{1-R}{R} \cdot \frac{\widehat{R}_{M L}}{1-\widehat{R}_{M L}} \leq F_{1-(1-\sqrt{1-\gamma}) / 2}(2 m, 2 n)\right] \\
& =P\left[\max \left\{T_{X}^{-1}\left(F_{(1+\sqrt[4]{1-\gamma}) / 2}(2 n-2,2)\right), T_{Y}^{-1}\left(F_{(1+\sqrt[4]{1-\gamma}) / 2}(2 m-2,2)\right)\right\} \leq \lambda\right. \\
& \leq \min \left\{T_{X}^{-1}\left(F_{(1-\sqrt[4]{1-\gamma}) / 2}(2 n-2,2)\right), T_{Y}^{-1}\left(F_{(1-\sqrt[4]{1-\gamma}) / 2}(2 m-2,2)\right)\right\} \\
& \left.\frac{1}{1+\frac{1-\widehat{R}_{M L}}{\widehat{R}_{M L}} F_{1-(1-\sqrt{1-\gamma}) / 2}(2 m, 2 n)} \leq R \leq \frac{1}{1+\frac{1-\widehat{R}_{M L}}{\widehat{R}_{M L}} F_{1-(1+\sqrt{1-\gamma}) / 2}(2 m, 2 n)}\right] \text {. }
\end{aligned}
$$

\subsection{Asymptotic confidence interval}

In this subsection, the asymptotic distribution of $\widehat{\theta}=(\widehat{\alpha}, \widehat{\beta}, \widehat{\lambda})$ and therefore the asymptotic distribution of $\widehat{R}$ are obtained. We denote the expected Fisher information matrix of $\theta=(\alpha, \beta, \lambda)$ as $J(\theta)=-E(I(\theta))$, where $I(\theta)=\left[I_{i j}\right], i, j=1,2,3$ is the observed information matrix. The observed Fisher information matrix has second partial derivatives of log-likelihood function as the entries, which can be obtained as 
follows:

$$
\begin{aligned}
& I_{11}=\frac{\partial^{2} \ell}{\partial \alpha^{2}}=-\frac{n}{\alpha^{2}}, \quad I_{22}=\frac{\partial^{2} \ell}{\partial \beta^{2}}=-\frac{m}{\beta^{2}}, \quad I_{12}=\frac{\partial^{2} \ell}{\partial \alpha \partial \beta}=0=I_{21}, \\
& I_{13}=\frac{\partial^{2} \ell}{\partial \alpha \partial \lambda}=\sum_{i=1}^{n} \frac{\left(R_{i}+1\right) e^{-\lambda / x_{i}^{2}}}{x_{i}^{2}\left(1-e^{-\lambda / x_{i}^{2}}\right)}=I_{31}, \\
& I_{23}=\frac{\partial^{2} \ell}{\partial \beta \partial \lambda}=\sum_{j=1}^{m} \frac{\left(S_{j}+1\right) e^{-\lambda / y_{j}^{2}}}{y_{j}^{2}\left(1-e^{-\lambda / y_{j}^{2}}\right)}=I_{32}, \\
& I_{33}=\frac{\partial^{2} \ell}{\partial \lambda^{2}}=-\frac{n+m}{\lambda^{2}}-\sum_{i=1}^{n} \frac{\left(\alpha\left(R_{i}+1\right)-1\right) e^{-\lambda / x_{i}^{2}}}{x_{i}^{4}\left(1-e^{-\lambda / x_{i}^{2}}\right)^{2}}-\sum_{j=1}^{m} \frac{\left(\beta\left(S_{j}+1\right)-1\right) e^{-\lambda / y_{j}^{2}}}{y_{j}^{4}\left(1-e^{-\lambda / y_{j}^{2}}\right)^{2}} .
\end{aligned}
$$

LEMMA 5. Suppose that $\left\{X_{1: n: N}, \ldots, X_{n: n: N}\right\}$ be a progressive censored sample from $\operatorname{IER}(\alpha, \lambda)$ with censored scheme $\left(N, n, R_{1}, \ldots, R_{n}\right)$. Then

(i). $E\left[\frac{e^{-\lambda / X_{i: n: N}^{2}}}{X_{i: n: N}^{2}\left(1-e^{\left.-\lambda / X_{i: n: N}^{2}\right)}\right.}\right]=\frac{\alpha C_{i-1}}{\lambda} \sum_{d=1}^{i} a_{i, d} \beta\left(2, \alpha \eta_{d}-1\right)\left[\psi\left(\alpha \eta_{d}+1\right)-\psi(2)\right]$,

(ii). $E\left[\frac{e^{-\lambda / X_{i: n: N}^{2}}}{X_{i: n: N}^{4}\left(1-e^{\left.-\lambda / X_{i: n: N}^{2}\right)^{2}}\right.}\right]$

$\frac{\alpha C_{i-1}}{\lambda^{2}} \sum_{d=1}^{i} a_{i, d} \beta\left(2, \alpha \eta_{d}-2\right)\left\{\left[\psi(2)-\psi\left(\alpha \eta_{d}\right)\right]^{2}+\psi^{\prime}(2)-\psi^{\prime}\left(\alpha \eta_{d}\right)\right\}$,

where $\psi(x)=\frac{d}{d x} \Gamma(x), \psi^{\prime}(x)=\frac{d^{2}}{d x^{2}} \Gamma(x), \beta(x, y)=\frac{\Gamma(x) \Gamma(y)}{\Gamma(x+y)}, \eta_{d}=n-d+1-\sum_{l=d}^{n} R_{l}$, $C_{i-1}=\prod_{d=1}^{i} \eta_{d}, a_{i, d}=\prod_{l=1, l \neq i}^{d} \frac{1}{\eta_{l}-\eta_{i}}$.

PROOF. It is known that if $\left\{X_{1: n: N}, \ldots, X_{n: n: N}\right\}$ is a progressive censored sample from $\operatorname{IER}(\alpha, \lambda)$, then the pdf of $X_{i: n: N}$ is (see [5]):

$$
\begin{aligned}
f_{X_{i: n: N}(x)} & =C_{i-1} \sum_{d=1}^{i} a_{i, d} f(x)[1-F(x)]^{\eta_{d}-1} \\
& =2 \alpha \lambda C_{i-1} \sum_{d=1}^{i} a_{i, d} \frac{e^{-\lambda / x^{2}}}{x^{3}}\left(1-e^{-\lambda / x^{2}}\right)^{\alpha \eta_{d}-1}
\end{aligned}
$$

(i). Using the table of the integrals from Gradshteyn and Ryzhik [10] (formulae 
4.253(1)), we have

$$
\begin{aligned}
E\left[\frac{e^{-\lambda / X_{i: n: N}^{2}}}{X_{i: n: N}^{2}\left(1-e^{-\lambda / X_{i: n: N}^{2}}\right)}\right] & =\int_{0}^{\infty} \frac{e^{-\lambda / x^{2}}}{x^{2}\left(1-e^{-\lambda / x^{2}}\right)} f_{X_{i: n: N}(x)} d x \\
& =2 \alpha \lambda C_{i-1} \sum_{d=1}^{i} a_{i, d} \int_{0}^{\infty} \frac{e^{-2 \lambda / x^{2}}}{x^{5}}\left(1-e^{-\lambda / x^{2}}\right)^{\alpha \eta_{d}-2} d x \\
& =-\frac{\alpha C_{i-1}}{\lambda} \sum_{d=1}^{i} a_{i, d} \int_{0}^{1} y \log (y)(1-y)^{\alpha \eta_{d}-2} d y \\
& =\frac{\alpha C_{i-1}}{\lambda} \sum_{d=1}^{i} a_{i, d} \beta\left(2, \alpha \eta_{d}-1\right)\left[\psi\left(\alpha \eta_{d}+1\right)-\psi(2)\right]
\end{aligned}
$$

(ii). Using the table of the integrals from Gradshteyn and Ryzhik [10] (formulae 4.261(21)), we have

$$
\begin{aligned}
& E\left[\frac{e^{-\lambda / X_{i: n: N}^{2}}}{X_{i: n: N}^{4}\left(1-e^{-\lambda / X_{i: n: N}^{2}}\right)^{2}}\right]=\int_{0}^{\infty} \frac{e^{-\lambda / x^{2}}}{x^{4}\left(1-e^{-\lambda / x^{2}}\right)^{2}} f_{X_{i: n: N}(x)} d x \\
& =2 \alpha \lambda C_{i-1} \sum_{d=1}^{i} a_{i, d} \int_{0}^{\infty} \frac{e^{-2 \lambda / x^{2}}}{x^{7}}\left(1-e^{-\lambda / x^{2}}\right)^{\alpha \eta_{d}-3} d x \\
& =\frac{\alpha C_{i-1}}{\lambda^{2}} \sum_{d=1}^{i} a_{i, d} \int_{0}^{1} y(\log (y))^{2}(1-y)^{\alpha \eta_{d}-3} d y \\
& =\frac{\alpha C_{i-1}}{\lambda^{2}} \sum_{d=1}^{i} a_{i, d} \beta\left(2, \alpha \eta_{d}-2\right)\left\{\left[\psi(2)-\psi\left(\alpha \eta_{d}\right)\right]^{2}+\psi^{\prime}(2)-\psi^{\prime}\left(\alpha \eta_{d}\right)\right\}
\end{aligned}
$$

With the previous lemma,

$$
\begin{aligned}
J_{11}= & \frac{n}{\alpha^{2}}, \quad J_{22}=\frac{m}{\beta^{2}}, \quad J_{12}=0=J_{21}, \\
J_{13}= & -\frac{\alpha}{\lambda} \sum_{i=1}^{n}\left(R_{i}+1\right) C_{i-1} \sum_{d=1}^{i} a_{i, d} \beta\left(2, \alpha \eta_{d}-1\right)\left[\psi\left(\alpha \eta_{d}+1\right)-\psi(2)\right]=J_{31}, \\
J_{23}= & -\frac{\beta}{\lambda} \sum_{j=1}^{m}\left(S_{j}+1\right) C_{j-1} \sum_{d=1}^{j} a_{j, d} \beta\left(2, \beta \eta_{d}-1\right)\left[\psi\left(\beta \eta_{d}+1\right)-\psi(2)\right]=J_{32}, \\
J_{33}= & \frac{\alpha}{\lambda^{2}} \sum_{i=1}^{n}\left(\alpha\left(R_{i}+1\right)-1\right) C_{i-1} \sum_{d=1}^{i} a_{i, d} \beta\left(2, \alpha \eta_{d}-2\right)\left\{\left[\psi(2)-\psi\left(\alpha \eta_{d}\right)\right]^{2}\right. \\
& \left.+\psi^{\prime}(2)-\psi^{\prime}\left(\alpha \eta_{d}\right)\right\}+\frac{\beta}{\lambda^{2}} \sum_{j=1}^{m}\left(\beta\left(S_{j}+1\right)-1\right) C_{j-1} \sum_{d=1}^{j} a_{j, d} \beta\left(2, \beta \eta_{d}-2\right) \\
& \times\left\{\left[\psi(2)-\psi\left(\beta \eta_{d}\right)\right]^{2}+\psi^{\prime}(2)-\psi^{\prime}\left(\beta \eta_{d}\right)\right\}+\frac{n+m}{\lambda^{2}} .
\end{aligned}
$$


Theorem 3. As $n \rightarrow \infty, m \rightarrow \infty$, and $n / m \rightarrow p$ then

$$
[\sqrt{n}(\widehat{\alpha}-\alpha) \sqrt{n}(\widehat{\beta}-\beta) \sqrt{m}(\widehat{\lambda}-\lambda)]^{T} \stackrel{D}{\longrightarrow} N_{3}\left(0, \mathbf{A}^{-\mathbf{1}}(\alpha, \beta, \lambda)\right),
$$

where $\mathbf{A}(\alpha, \beta, \lambda)$ and $\mathbf{A}^{-1}(\alpha, \beta, \lambda)$ are symmetric matrices and

$$
\mathbf{A}(\alpha, \beta, \lambda)=\left(\begin{array}{ccc}
\frac{J_{11}}{n} & 0 & \frac{J_{13}}{\sqrt{n m}} \\
& \frac{J_{22}}{n} & \frac{J_{23}}{\sqrt{n m}} \\
& & \frac{J_{33}}{m}
\end{array}\right), \mathbf{A}^{-1}(\alpha, \beta, \lambda)=\frac{1}{|\mathbf{A}(\alpha, \beta, \lambda)|}\left(\begin{array}{ccc}
\frac{b_{11}}{n m} & \frac{b_{12}}{n m} & \frac{b_{13}}{n \sqrt{n m}} \\
& \frac{b_{22}}{n m} & \frac{b_{23}}{n \sqrt{n m}} \\
& \frac{b_{33}}{n^{2}}
\end{array}\right),
$$

and $|\mathbf{A}(\alpha, \beta, \lambda)|=\frac{1}{n^{2} m}\left(J_{11} J_{22} J_{33}-J_{11} J_{23}^{2}-J_{13}^{2} J_{22}\right)$,

$$
\begin{aligned}
& b_{11}=J_{22} J_{33}-J_{23}^{2}, \quad b_{12}=J_{13} J_{23}, \quad b_{13}=-J_{13} J_{22}, \\
& b_{22}=J_{11} J_{33}-J_{13}^{2}, \quad b_{23}=-J_{11} J_{23}, \quad b_{33}=J_{11} J_{22} \text {. }
\end{aligned}
$$

PROOF. By using the asymptotic properties of MLEs and the multivariate central limit theorem,

$$
[(\widehat{\alpha}-\alpha)(\widehat{\beta}-\beta)(\widehat{\lambda}-\lambda)]^{T} \stackrel{D}{\longrightarrow} N_{3}\left(0, \mathbf{J}^{-\mathbf{1}}(\alpha, \beta, \lambda)\right),
$$

where $\mathbf{J}(\alpha, \beta, \lambda)$ and $\mathbf{J}^{-\mathbf{1}}(\alpha, \beta, \lambda)$ are symmetric matrices and

$$
\mathbf{J}(\alpha, \beta, \lambda)=\left(\begin{array}{ccc}
J_{11} & 0 & J_{13} \\
& J_{22} & J_{23} \\
& & J_{33}
\end{array}\right), \mathbf{J}^{-1}(\alpha, \beta, \lambda)=\frac{1}{|\mathbf{J}(\alpha, \beta, \lambda)|}\left(\begin{array}{rrr}
b_{11} & b_{12} & b_{13} \\
& b_{22} & b_{23} \\
& b_{33}
\end{array}\right),
$$

and $|\mathbf{J}(\alpha, \beta, \lambda)|=n^{2} m|\mathbf{A}(\alpha, \beta, \lambda)|$. Let $\mathbf{C}=\left(\begin{array}{ccc}\sqrt{n} & 0 & 0 \\ 0 & \sqrt{n} & 0 \\ 0 & 0 & \sqrt{m}\end{array}\right)$ then

$$
\mathbf{C}[(\widehat{\alpha}-\alpha)(\widehat{\beta}-\beta)(\widehat{\lambda}-\lambda)]^{T} \stackrel{D}{\longrightarrow} N_{3}\left(0, \mathbf{C J}^{-\mathbf{1}}(\alpha, \beta, \lambda) \mathbf{C}^{\mathbf{T}}\right),
$$

where $\mathbf{C}[(\widehat{\alpha}-\alpha)(\widehat{\beta}-\beta)(\widehat{\lambda}-\lambda)]^{T}=[\sqrt{n}(\widehat{\alpha}-\alpha) \sqrt{n}(\widehat{\beta}-\beta) \sqrt{m}(\widehat{\lambda}-\lambda)]^{T}$ and

$$
\mathbf{C J}^{-\mathbf{1}}(\alpha, \beta, \lambda) \mathbf{C}^{\mathbf{T}}=\frac{1}{|\mathbf{A}(\alpha, \beta, \lambda)|}\left(\begin{array}{ccc}
\frac{b_{11}}{n m} & \frac{b_{12}}{n m} & \frac{b_{13}}{n \sqrt{n m}} \\
& \frac{b_{22}}{n m} & \frac{b_{23}}{n \sqrt{n m}} \\
& \frac{b_{33}}{n^{2}}
\end{array}\right) .
$$

Therefore, the result follows. 
THEOREM 4. As $n \rightarrow \infty, m \rightarrow \infty$, and $n / m \rightarrow p$ then

$$
\sqrt{n}(\widehat{R}-R) \stackrel{D}{\longrightarrow} N(0, B),
$$

where

$$
B=\frac{1}{(n m)|\mathbf{A}(\alpha, \beta, \lambda)|(\alpha+\beta)^{4}}\left[\alpha^{2} b_{22}+\beta^{2} b_{11}-2 \alpha \beta b_{12}\right] .
$$

ProOF. On using Theorem 3 and applying delta method, we can describe the asymptotic distribution of $\widehat{R}=g(\widehat{\alpha}, \widehat{\beta}, \widehat{\lambda})$, where $g(\alpha, \beta, \lambda)=\frac{\alpha}{\alpha+\beta}$ as the following

$$
\sqrt{n}(\widehat{R}-R) \stackrel{D}{\longrightarrow} N(0, B),
$$

where $B=\mathbf{b}^{\mathbf{T}} \mathbf{A}^{-\mathbf{1}}(\alpha, \beta, \lambda) \mathbf{b}$, with $\mathbf{b}=\left[\begin{array}{lll}\frac{\partial g}{\partial \alpha} & \frac{\partial g}{\partial \beta} & \frac{\partial g}{\partial \lambda}\end{array}\right]^{T}=\frac{1}{(\alpha+\beta)^{2}}\left[\begin{array}{lll}\beta & -\alpha & 0\end{array}\right]^{T}$, and $\mathbf{A}^{-1}(\alpha, \beta, \lambda)$ is defined in Theorem 3. Therefore

$$
B=\mathbf{b}^{\mathbf{T}} \mathbf{A}^{-\mathbf{1}}(\alpha, \beta, \lambda) \mathbf{b}=\frac{1}{(n m)|\mathbf{A}(\alpha, \beta, \lambda)|(\alpha+\beta)^{4}}\left[\alpha^{2} b_{22}+\beta^{2} b_{11}-2 \alpha \beta b_{12}\right] .
$$

Thus, the proof is obtained.

From Theorem 4, we construct the asymptotic confidence interval of $R$. Using the MLEs of $\alpha, \beta$ and $\lambda, B$ can be estimated. Therefore, a $100(1-\gamma) \%$ asymptotic confidence interval for $R$ can be presented of the form,

$$
\left(\widehat{R}-z_{1-\frac{\gamma}{2}} \frac{\sqrt{\widehat{B}}}{\sqrt{n}}, \widehat{R}+z_{1-\frac{\gamma}{2}} \frac{\sqrt{\widehat{B}}}{\sqrt{n}}\right),
$$

where $z_{\gamma}$ is $100 \gamma$-th percentile of $N(0,1)$.

\subsection{Confidence interval based on bootstrap procedures}

Since the sampling distribution of $\widehat{R}$ is not available if $\lambda$ is unknown, the bootstrapping method can be an alternative instead of the method described in previous subsection to develop an approximated confidence interval of the parameter $R$. Also, it is evident that the confidence interval based on the asymptotic results do not perform very well for small sample size. For this, we propose two confidence intervals based on the non-parametric bootstrap methods: (i) percentile bootstrap method (we call it Boot-p) based on the original idea of Efron [9] and (ii) bootstrap-t method (we refer it as Boot-t) based on the idea of Hall [12]. 


\section{(i) Boot-p Method}

1. Generate a bootstrap sample of size $n,\left\{x_{1}^{*}, \ldots, x_{n}^{*}\right\}$ from $\left\{x_{1}, \ldots, x_{n}\right\}$ and generate a bootstrap sample of size $m,\left\{y_{1}^{*}, \ldots, y_{m}^{*}\right\}$ from $\left\{y_{1}, \ldots, y_{m}\right\}$. Based on $\left\{x_{1}^{*}, \ldots, x_{n}^{*}\right\}$ and $\left\{y_{1}^{*}, \ldots, y_{m}^{*}\right\}$, compute the bootstrap estimate of $R$ say $\widehat{R}^{*}$, using (6).

2. Repeat 1 NBOOT times.

3. Let $G^{*}(x)=P\left(\widehat{R}^{*} \leq x\right)$ be the cumulative distribution function of $\widehat{R}^{*}$. Define $\widehat{R}_{B p}(x)=G^{*-1}(x)$ for a given $x$. The approximate $100(1-\gamma) \%$ confidence interval of $R$ is given by

$$
\left(\widehat{R}_{B p}\left(\frac{\gamma}{2}\right), \widehat{R}_{B p}\left(1-\frac{\gamma}{2}\right)\right)
$$

\section{(ii) Boot-t Method}

1. From the samples $\left\{x_{1}, \ldots, x_{n}\right\}$ and $\left\{y_{1}, \ldots, y_{m}\right\}$, compute $\widehat{R}$.

2. Same as in Boot-p method, first generate bootstrap sample $\left\{x_{1}^{*}, \ldots, x_{n}^{*}\right\}$ and $\left\{y_{1}^{*}, \ldots, y_{m}^{*}\right\}$ and then compute $\widehat{R}^{*}$, the bootstrap estimate of $R$. Also, compute the statistic:

$$
T^{*}=\frac{\sqrt{n}\left(\widehat{R}^{*}-\widehat{R}\right)}{\sqrt{V\left(\widehat{R}^{*}\right)}}
$$

Compute $V\left(\widehat{R}^{*}\right)$ using Theorem 4 .

3. Repeat steps 1 and 2 NBOOT times.

4. Let $H(x)=P\left(T^{*} \leq x\right)$ be the cumulative distribution function of $T^{*}$. For a given $x$, define

$$
\widehat{R}_{B t}(x)=\widehat{R}+n^{-\frac{1}{2}} H^{-1}(x) \sqrt{V(\widehat{R})} .
$$

The approximate $100(1-\gamma) \%$ confidence interval of $R$ is given by:

$$
\left(\widehat{R}_{B t}\left(\frac{\gamma}{2}\right), \widehat{R}_{B t}\left(1-\frac{\gamma}{2}\right)\right) \text {. }
$$




\section{BAYES ESTIMATION OF $R$}

In this section, we develop the Bayesian inference of $R$ under the assumption that the parameters $\alpha, \beta$ and $\lambda$ are random variables. We mainly discuss the Bayes estimates and the associated credible intervals of $R$. It is assumed that $\alpha, \beta$ and $\lambda$ have independent gamma priors with the PDF's:

$$
\begin{array}{lll}
\pi_{1}(\alpha) \propto \alpha^{a_{1}-1} e^{-b_{1} \alpha}, & & \alpha>0, a_{1}>0, b_{1}>0, \\
\pi_{2}(\beta) \propto \beta^{a_{2}-1} e^{-b_{2} \beta}, & & \beta>0, a_{2}>0, b_{2}>0, \\
\pi_{3}(\lambda) \propto \lambda^{a_{3}-1} e^{-b_{3} \lambda}, & & \lambda>0, a_{3}>0, b_{3}>0,
\end{array}
$$

respectively. Based on the observed sample, the joint posterior density function of $\alpha$, $\beta$ and $\lambda$ is

$$
\pi(\alpha, \beta, \lambda \mid \text { data })=\frac{L(\text { data } \mid \alpha, \beta, \lambda) \pi_{1}(\alpha) \pi_{2}(\beta) \pi_{3}(\lambda)}{\int_{0}^{\infty} \int_{0}^{\infty} \int_{0}^{\infty} L(\text { data } \mid \alpha, \beta, \lambda) \pi_{1}(\alpha) \pi_{2}(\beta) \pi_{3}(\lambda) d \alpha d \beta d \lambda}
$$

From (11), it is obvious that the Bayes estimate will not be analytically obtained. Consequently, we adopt the Gibbs and Metropolis sampling techniques to compute the Bayes estimate of $R$ and the corresponding credible interval of $R$. The posterior pdfs of $\alpha$ and $\beta$ are as follows:

$$
\begin{aligned}
& \alpha \mid \beta, \lambda, \text { data } \sim \Gamma\left(n+a_{1}, b_{1}+\sum_{i=1}^{n}\left(R_{i}+1\right) \log \left(\frac{1}{1-e^{-\lambda / x_{i}^{2}}}\right)\right), \\
& \beta \mid \alpha, \lambda, \text { data } \sim \Gamma\left(m+a_{2}, b_{2}+\sum_{j=1}^{m}\left(S_{j}+1\right) \log \left(\frac{1}{1-e^{-\lambda / y_{j}^{2}}}\right)\right),
\end{aligned}
$$

and

$$
\begin{aligned}
\pi(\lambda \mid \alpha, \beta, \text { data }) & \propto \lambda^{n+m+a_{3}-1} \exp \left\{-\lambda\left(\sum_{i=1}^{n} \frac{1}{x_{i}^{2}}+\sum_{j=1}^{m} \frac{1}{y_{j}^{2}}+b_{3}\right)\right\} \\
& \times\left(\prod_{i=1}^{n}\left(1-e^{-\lambda / x_{i}^{2}}\right)^{-1}\right)\left(\prod_{j=1}^{m}\left(1-e^{-\lambda / y_{j}^{2}}\right)^{-1}\right) .
\end{aligned}
$$

The posterior pdf of $\lambda$ is not known. So to generate random number from the posterior pdf of $\lambda$, we use the Metropolis-Hastings method. Therefore, the algorithm of Gibbs sampling is as follows:

1. Start with an initial guess $\left(\alpha_{(0)}, \beta_{(0)}, \lambda_{(0)}\right)$.

2. Set $t=1$.

3. Generate $\lambda_{(t)}$ from $\pi\left(\lambda \mid \alpha_{(t-1)}, \beta_{(t-1)}\right.$, data). 
4. Generate $\alpha_{(t)}$ from $\Gamma\left(n+a_{1}, b_{1}+\sum_{i=1}^{n}\left(R_{i}+1\right) \log \left(\frac{1}{1-e^{-\lambda(t-1)} / x_{i}^{2}}\right)\right)$.

5. Generate $\beta_{(t)}$ from $\Gamma\left(m+a_{2}, b_{2}+\sum_{j=1}^{m}\left(S_{j}+1\right) \log \left(\frac{1}{1-e^{-\lambda(t-1)} / y_{j}^{2}}\right)\right)$.

6. Compute $R_{(t)}=\frac{\alpha_{(t)}}{\alpha_{(t)}+\beta_{(t)}}$.

7. Set $t=t+1$.

8. Repeat steps 3-7, $T$ times.

Now the approximate posterior mean, and posterior variance of $\mathrm{R}$ become

$$
\widehat{E}(R \mid \text { data })=\frac{1}{T} \sum_{t=1}^{T} R_{(t)}, \widehat{\operatorname{Var}}(R \mid \text { data })=\frac{1}{T} \sum_{t=1}^{T}\left(R_{(t)}-\widehat{E}(R \mid \text { data })\right)^{2} .
$$

Based on $T$ and $R$ values, using the method proposed by Chen and Shao [8], a $100(1-\gamma) \%$ HPD (Highest Posterior Density) credible interval can be constructed as $\left(R_{\left[\frac{\gamma}{2} T\right]}, R_{\left[\left(1-\frac{\gamma}{2}\right) T\right]}\right)$, where $R_{\left[\frac{\gamma}{2} T\right]}$ and $R_{\left[\left(1-\frac{\gamma}{2}\right) T\right]}$ are the $\left[\frac{\gamma}{2} T\right]$-th smallest integer and the $\left[\left(1-\frac{\gamma}{2}\right) T\right]$-th smallest integer of $\left\{R_{t}=1,2, \ldots, T\right\}$, respectively.

Here we obtain the Bayes estimation of $R$ under the assumptions that the parameters $\alpha$ and $\beta$ are random variables and the parameter $\lambda$ is known. It is assumed that $\alpha$ and $\beta$ have independent gamma priors with parameters $\left(a_{1}, b_{1}\right)$ and $\left(a_{2}, b_{2}\right)$, respectively. The posterior pdf's of $\alpha$ and $\beta$ can be shown to be $\Gamma\left(n+a_{1}, b_{1}+A_{1}(\mathbf{x})\right)$ and $\Gamma\left(m+a_{2}, b_{2}+A_{2}(\mathbf{y})\right)$, respectively, where, $\left.A_{1}(\mathbf{x})\right)=T_{1} /(2 \alpha)$ and $\left.A_{2}(\mathbf{y})\right)=T_{2} /(2 \beta)$. Since the priors $\alpha$ and $\beta$ are independent, the posterior pdf of $R$ becomes

$$
f_{R}(r)=S \frac{r^{n+a_{1}-1}(1-r)^{m+a_{2}-1}}{\left[r\left(b_{1}+A_{1}(\mathbf{x})\right)+(1-r)\left(b_{2}+A_{2}(\mathbf{y})\right)\right]^{n+m+a_{1}+a_{2}}}, \quad 0<r<1,
$$

where

$$
S=\frac{\Gamma\left(n+m+a_{1}+a_{2}\right)}{\Gamma\left(n+a_{1}\right) \Gamma\left(m+a_{2}\right)}\left(b_{1}+A_{1}(\mathbf{x})\right)^{n+a_{1}}\left(b_{2}+A_{2}(\mathbf{y})\right)^{m+a_{2}} .
$$

Since the Bayes estimate of $R$ under the squared error loss function can not be obtained analytically, we approximate it via the method of Lindley [19]. Alternatively, using the approximation of Lindley [19] and following the approach of Ahmad et al. [2], it can be seen that the approximate Bayes estimate of $R$, say $\widehat{R}_{B S}$, under the squared error loss function is

$$
\widehat{R}_{B S}=\tilde{R}\left\{1+\frac{\tilde{\alpha} \tilde{R}^{2}}{\tilde{\beta}^{2}\left(m+a_{2}-1\right)\left(n+b_{1}-1\right)}\left[\tilde{\alpha}\left(n+a_{1}-1\right)-\tilde{\beta}\left(m+a_{2}-2\right)\right]\right\},
$$


where

$$
\tilde{R}=\frac{\tilde{\alpha}}{\tilde{\alpha}+\tilde{\beta}}, \quad \tilde{\alpha}=\frac{n+a_{1}-1}{b_{1}+A_{1}(\mathbf{x})}, \quad \text { and } \quad \tilde{\beta}=\frac{m+a_{2}-1}{b_{2}+A_{2}(\mathbf{y})} .
$$

The $100(1-\gamma) \%$ Bayesian interval for $R$ is given by $(L, U)$, where $L$ and $U$ are the lower and upper bounds, respectively, satisfying

$$
P[R \leq L \mid \text { data }]=\frac{\gamma}{2}, \text { and } P[R \leq U \mid \text { data }]=1-\frac{\gamma}{2} .
$$

\section{DATA ANALYSIS AND COMPARISON STUDY}

In this section, we present some results based on Monte Carlo simulations and real data to compare the performance of the different methods described in the preceding sections.

\subsection{Numerical experiments and discussions}

In this subsection, the Monte Carlo simulation is conducted to compare the performance of MLE, UMVUE and Bayes estimator under different progressive censoring schemes. We compare the performances of the different estimators in terms of biases, and mean squares errors (MSE). We also compare different confidence intervals, namely the asymptotic confidence intervals, two bootstrap confidence intervals and the HPD credible intervals in terms of the average confidence lengths, and coverage percentages. We use different parameter values, different hyper parameters and different sampling schemes. We used three sets of parameter values $\Theta_{1}=(\alpha=2, \beta=2, \lambda=0.5), \Theta_{2}=(\alpha=2, \beta=2, \lambda=1.5)$ and $\Theta_{3}=(\alpha=2, \beta=2, \lambda=2.5)$ mainly to compare the MLEs and different Bayes estimators. For computing the Bayes estimators and HPD credible intervals, we assume 3 priors as follows:

$\begin{array}{llll}\text { Prior 1: } & a_{j}=0, & b_{j}=0, & j=1,2,3, \\ \text { Prior 2: } & a_{j}=1, & b_{j}=2, & j=1,2,3, \\ \text { Prior 3: } & a_{j}=2, & b_{j}=3, & j=1,2,3 .\end{array}$

Prior 1 is the non-informative gamma prior and Priors 2 and 3 are informative gamma priors. We also use three censoring schemes as given in Table I.

For different parameter values, different censoring schemes and different priors, we report the average biases, and MSE of the MLE and Bayes estimates of $R$ over 1000 
Table I. Censoring schemes.

\begin{tabular}{|ccc|}
\hline & $(n, N)$ & C.S. \\
\hline$r_{1}$ & $(10,30)$ & $(0,0,0,0,0,0,0,0,0,20)$ \\
$r_{2}$ & $(10,30)$ & $(20,0,0,0,0,0,0,0,0,0)$ \\
$r_{3}$ & $(10,30)$ & $(2,2,2,2,2,2,2,2,2,2)$ \\
\hline
\end{tabular}

Table II. Biases and MSE of the MLE and Bayes estimates of $R$.

\begin{tabular}{|c|c|cc|c|c|c|c|cc|}
\hline$\Theta_{j}$ & C.S & \multicolumn{2}{|c|}{ MLE } & \multicolumn{2}{|c|}{ Prior } & \multicolumn{2}{|c|}{ Prior } & \multicolumn{2}{|c|}{ Prior3 } \\
\cline { 3 - 9 } & & $\mid$ Bias & MSE & $\mid$ Bias & MSE & $\mid$ Bias & MSE & Bias & MSE \\
\hline \multirow{5}{*}{$\Theta_{1}$} & $\left(r_{1}, r_{1}\right)$ & 0.0094 & 0.0200 & 0.0045 & 0.0158 & 0.0015 & 0.0141 & 0.0001 & 0.0128 \\
& $\left(r_{2}, r_{2}\right)$ & 0.0025 & 0.0225 & 0.0021 & 0.0160 & 0.0007 & 0.0133 & 0.0006 & 0.0116 \\
& $\left(r_{3}, r_{3}\right)$ & 0.0029 & 0.0229 & 0.0021 & 0.0151 & 0.0009 & 0.0143 & 0.0009 & 0.0122 \\
& $\left(r_{1}, r_{2}\right)$ & 0.0022 & 0.0215 & 0.0018 & 0.0155 & 0.0014 & 0.0137 & 0.0008 & 0.0119 \\
& $\left(r_{1}, r_{3}\right)$ & 0.0073 & 0.0219 & 0.0049 & 0.0156 & 0.0039 & 0.0140 & 0.0038 & 0.0121 \\
& $\left(r_{2}, r_{3}\right)$ & 0.0055 & 0.0218 & 0.0018 & 0.0163 & 0.0013 & 0.0141 & 0.0004 & 0.0123 \\
\hline \multirow{5}{*}{$\Theta_{2}$} & $\left(r_{1}, r_{1}\right)$ & 0.0079 & 0.0230 & 0.0028 & 0.0156 & 0.0022 & 0.0134 & 0.0006 & 0.0133 \\
& $\left(r_{2}, r_{2}\right)$ & 0.0070 & 0.0222 & 0.0042 & 0.0159 & 0.0018 & 0.0131 & 0.0003 & 0.0108 \\
& $\left(r_{3}, r_{3}\right)$ & 0.0046 & 0.0222 & 0.0010 & 0.0160 & 0.0009 & 0.0140 & 0.0007 & 0.0126 \\
& $\left(r_{1}, r_{2}\right)$ & 0.0085 & 0.0216 & 0.0075 & 0.0160 & 0.0011 & 0.0143 & 0.0004 & 0.0126 \\
& $\left(r_{1}, r_{3}\right)$ & 0.0079 & 0.0217 & 0.0053 & 0.0154 & 0.0047 & 0.0139 & 0.0022 & 0.0127 \\
& $\left(r_{2}, r_{3}\right)$ & 0.0095 & 0.0205 & 0.0057 & 0.0162 & 0.0054 & 0.0136 & 0.0033 & 0.0131 \\
\hline \multirow{5}{*}{$\Theta_{3}$} & $\left(r_{1}, r_{1}\right)$ & 0.0035 & 0.0210 & 0.0023 & 0.0162 & 0.0013 & 0.0136 & 0.0011 & 0.0133 \\
& $\left(r_{2}, r_{2}\right)$ & 0.0044 & 0.0222 & 0.0033 & 0.0163 & 0.0025 & 0.0134 & 0.0013 & 0.0119 \\
& $\left(r_{3}, r_{3}\right)$ & 0.0027 & 0.0218 & 0.0026 & 0.0148 & 0.0011 & 0.0143 & 0.0008 & 0.0124 \\
& $\left(r_{1}, r_{2}\right)$ & 0.0089 & 0.0211 & 0.0047 & 0.0156 & 0.0043 & 0.0138 & 0.0018 & 0.0132 \\
& $\left(r_{1}, r_{3}\right)$ & 0.0063 & 0.0215 & 0.0061 & 0.0168 & 0.0038 & 0.0137 & 0.0027 & 0.0129 \\
& $\left(r_{2}, r_{3}\right)$ & 0.0042 & 0.0220 & 0.0035 & 0.0160 & 0.0015 & 0.0135 & 0.0003 & 0.0124 \\
\hline
\end{tabular}

replications. The results are reported in Table II. In our simulation experiments for the bootstrap method, we have computed the confidence intervals based on 250 resampling. The Bayes estimates and the corresponding credible intervals are based on 1000 sampling, namely $T=1000$.

From Table II, we observe that the MLE compares very well with the Bayes estimator in terms of biases and MSEs. Comparing the two Bayes estimators based on two informative gamma priors clearly shows that the Bayes estimators based on prior 3 perform better than the Bayes estimators based on prior 2, in terms of both biases and MSEs. The Bayes estimators based on both priors perform better than the ones obtained using the non-informative prior 1.

We also computed the $95 \%$ confidence intervals for $R$ based on the asymptotic distribution of the MLE. Furthermore, the Boot-p, Boot-t confidence intervals and the HPD credible intervals are computed. In Table III, we presented the average 
Table III. Average confidence/credible length and coverage percentage for estimators of $R$.

\begin{tabular}{|c|c|c|c|c|c|c|}
\hline \multicolumn{7}{|c|}{$\Theta_{1}$} \\
\hline \multirow[t]{2}{*}{ C.S } & \multirow[t]{2}{*}{ Boot-t } & \multirow[t]{2}{*}{ Boot-p } & \multirow[t]{2}{*}{ MLE } & \multicolumn{3}{|c|}{ Bayes } \\
\hline & & & & Prior1 & Prior2 & Prior3 \\
\hline$\left(r_{1}, r_{1}\right)$ & $0.5505(0.950)$ & $0.4740(0.944)$ & $0.4554(0.948)$ & $0.4413(0.947)$ & $0.4304(0.950)$ & $0.4239(0.949)$ \\
\hline$\left(r_{2}, r_{2}\right)$ & $0.5801(0.958)$ & $0.4812(0.952)$ & $0.4511(0.949)$ & $0.4235(0.940)$ & $0.4202(0.954)$ & $0.4171(0.956)$ \\
\hline$\left(r_{3}, r_{3}\right)$ & $0.5802(0.959)$ & $0.4757(0.948)$ & $0.4549(0.941)$ & $0.4490(0.944)$ & $0.4233(0.941)$ & $0.4192(0.956)$ \\
\hline$\left(r_{1}, r_{2}\right)$ & $0.5681(0.957)$ & $0.4700(0.942)$ & $0.4477(0.943)$ & $0.4470(0.947)$ & $0.4269(0.947)$ & $0.4186(0.950)$ \\
\hline$\left(r_{1}, r_{3}\right)$ & $0.5595(0.956)$ & $0.4807(0.940)$ & $0.4583(0.954)$ & $0.4389(0.945)$ & $0.4380(0.954)$ & $0.4261(0.952)$ \\
\hline$\left(r_{2}, r_{3}\right)$ & $0.5794(0.957)$ & $0.4810(0.944)$ & $0.4569(0.943)$ & $0.4344(0.942)$ & $0.4239(0.948)$ & $0.4142(0.946)$ \\
\hline \multicolumn{7}{|c|}{$\Theta_{2}$} \\
\hline \multirow[t]{2}{*}{ C.S } & \multirow[t]{2}{*}{ Boot-t } & \multirow[t]{2}{*}{ Boot-p } & \multirow[t]{2}{*}{ MLE } & \multicolumn{3}{|c|}{ Bayes } \\
\hline & & & & Prior1 & Prior2 & Prior3 \\
\hline$\left(r_{1}, r_{1}\right)$ & $0.5663(0.958)$ & $0.4634(0.949)$ & $0.4564(0.956)$ & $0.4479(0.954)$ & $0.4462(0.941)$ & $0.4232(0.955)$ \\
\hline$\left(r_{2}, r_{2}\right)$ & $0.5627(0.956)$ & $0.4868(0.941)$ & $0.4483(0.947)$ & $0.4243(0.953)$ & $0.4128(0.942)$ & $0.4068(0.943)$ \\
\hline$\left(r_{3}, r_{3}\right)$ & $0.5602(0.958)$ & $0.4778(0.955)$ & $0.4563(0.941)$ & $0.4399(0.945)$ & $0.4369(0.955)$ & $0.4225(0.947)$ \\
\hline$\left(r_{1}, r_{2}\right)$ & $0.5636(0.956)$ & $0.4681(0.946)$ & $0.4571(0.954)$ & $0.4566(0.942)$ & $0.4480(0.950)$ & $0.4464(0.949)$ \\
\hline$\left(r_{1}, r_{3}\right)$ & $0.5574(0.953)$ & $0.4889(0.951)$ & $0.4581(0.950)$ & $0.4335(0.953)$ & $0.4314(0.949)$ & $0.4261(0.948)$ \\
\hline$\left(r_{2}, r_{3}\right)$ & $0.5462(0.952)$ & $0.4825(0.948)$ & $0.4547(0.945)$ & $0.4325(0.947)$ & $0.4318(0.942)$ & $0.3901(0.940)$ \\
\hline \multicolumn{7}{|c|}{$\Theta_{3}$} \\
\hline \multirow[t]{2}{*}{ C.S } & \multirow[t]{2}{*}{ Boot-t } & \multirow[t]{2}{*}{ Boot-p } & \multirow[t]{2}{*}{ MLE } & \multicolumn{3}{|c|}{ Bayes } \\
\hline & & & & Prior1 & Prior2 & Prior3 \\
\hline$\left(r_{1}, r_{1}\right)$ & $0.5562(0.958)$ & $0.4770(0.957)$ & $0.4459(0.942)$ & $0.4445(0.951)$ & $0.4430(0.952)$ & $0.4233(0.951)$ \\
\hline$\left(r_{2}, r_{2}\right)$ & $0.5468(0.956)$ & $0.4806(0.954)$ & $0.4478(0.947)$ & $0.4230(0.943)$ & $0.4141(0.953)$ & $0.4137(0.940)$ \\
\hline$\left(r_{3}, r_{3}\right)$ & $0.5557(0.954)$ & $0.4824(0.952)$ & $0.4545(0.942)$ & $0.4337(0.952)$ & $0.4325(0.950)$ & $0.4235(0.953)$ \\
\hline$\left(r_{1}, r_{2}\right)$ & $0.5687(0.952)$ & $0.4874(0.943)$ & $0.4562(0.946)$ & $0.4518(0.951)$ & $0.4460(0.945)$ & $0.4424(0.946)$ \\
\hline$\left(r_{1}, r_{3}\right)$ & $0.5483(0.957)$ & $0.4942(0.950)$ & $0.4570(0.948)$ & $0.4429(0.950)$ & $0.4329(0.949)$ & $0.4252(0.950)$ \\
\hline$\left(r_{2}, r_{3}\right)$ & $0.5672(0.958)$ & $0.4879(0.940)$ & $0.4399(0.953)$ & $0.4341(0.951)$ & $0.4338(0.946)$ & $0.4134(0.945)$ \\
\hline
\end{tabular}

confidence or credible lengths, and the corresponding coverage percentages. The nominal level for the confidence intervals or the credible intervals is 0.95 in each case. From Table III, we observe that the bootstrap confidence intervals are wider than the other confidence interval. We also observe that the HPD intervals provide the smallest average confidence credible lengths for different censoring schemes, and for different parameter values. The asymptotic confidence interval MLE is the second best confidence intervals. It is also observed that Boot-p confidence intervals perform better than the Boot-t confidence intervals. From Table III, it is evident that the Boot-t credible intervals provide the most coverage probabilities in most cases considered. Comparing the two HPD credible intervals based on two informative gamma priors clearly shows that the HPD credible intervals based on prior 3 perform smaller than the HPD credible interval based on prior 2. The HPD credible intervals based on both priors perform smaller than the ones obtained using the non-informative prior 1 .

Now we consider the case when the common scale parameter $\lambda$ is known. In this case, we obtain the MLE and UMVUE of $R$ using (10) and (9), respectively. Since we have no prior information on $R$, we prefer to use the non-informative prior i.e $a_{1}=$ 
$b_{1}=a_{2}=b_{2}=0$ to compute Bayes estimates. Applying the same prior distributions, the Lindley approximation Bayes estimates are computed using (12). Also, using (13) the Bayesian interval based on Lindley approximation is obtained. We report the average biases and MSEs based on 1000 replications. The results are reported in Table IV. From Table IV, comparing the MLE, UMVUE and Bayes estimator, we observe that MLE provides the smallest biases and MSEs and the UMVUEs are the best second estimators.

Table IV. Biases and MSE of the MLE, UMVUE and Bayes estimators of $R$ and average confidence length and coverage percentage when $\lambda$ is known.

\begin{tabular}{|c|c|c|c|c|c|c|c|}
\hline \multicolumn{8}{|c|}{$\lambda=0.5$} \\
\hline \multirow[t]{2}{*}{ C.S } & \multicolumn{2}{|c|}{ MLE } & \multicolumn{2}{|c|}{ UMVUE } & \multicolumn{2}{|c|}{ Lindley } & \multirow[t]{2}{*}{ Lindley } \\
\hline & Bias & MSE & Bias | & MSE & Bias | & MSE & \\
\hline$\left(r_{1}, r_{1}\right)$ & 0.0002 & 0.0112 & 0.0012 & 0.0116 & 0.0022 & 0.0135 & $0.4117(0.951)$ \\
\hline$\left(r_{2}, r_{2}\right)$ & 0.0001 & 0.0117 & 0.0008 & 0.0124 & 0.0043 & 0.0125 & $0.4171(0.952)$ \\
\hline$\left(r_{3}, r_{3}\right)$ & 0.0020 & 0.0112 & 0.0040 & 0.0115 & 0.0045 & 0.0131 & $0.4296(0.952)$ \\
\hline$\left(r_{1}, r_{2}\right)$ & 0.0019 & 0.0124 & 0.0032 & 0.0125 & 0.0056 & 0.0135 & $0.4248(0.947)$ \\
\hline$\left(r_{1}, r_{3}\right)$ & 0.0016 & 0.0114 & 0.0019 & 0.0124 & 0.0023 & 0.0125 & $0.4257(0.943)$ \\
\hline$\left(r_{2}, r_{3}\right)$ & 0.0005 & 0.0117 & 0.0007 & 0.0124 & 0.0051 & 0.0133 & $0.4242(0.946)$ \\
\hline \multicolumn{8}{|c|}{$\lambda=1.5$} \\
\hline \multirow[t]{2}{*}{ C.S } & \multicolumn{2}{|c|}{ MLE } & \multicolumn{2}{|c|}{ Lindley } & \multicolumn{2}{|c|}{ UMVUE } & \multirow[t]{2}{*}{ Lindley } \\
\hline & Bias & MSE & \begin{tabular}{|l|} 
Bias \\
\end{tabular} & MSE & Bias & MSE & \\
\hline$\left(r_{1}, r_{1}\right)$ & 0.0006 & 0.0111 & 0.0049 & 0.0117 & 0.0090 & 0.0129 & $0.4136(0.951)$ \\
\hline$\left(r_{2}, r_{2}\right)$ & 0.0026 & 0.0111 & 0.0027 & 0.0124 & 0.0036 & 0.0125 & $0.3975(0.942)$ \\
\hline$\left(r_{3}, r_{3}\right)$ & 0.0012 & 0.0112 & 0.0036 & 0.0118 & 0.0081 & 0.0122 & $0.4054(0.944)$ \\
\hline$\left(r_{1}, r_{2}\right)$ & 0.0014 & 0.0118 & 0.0040 & 0.0120 & 0.0066 & 0.0128 & $0.4197(0.949)$ \\
\hline$\left(r_{1}, r_{3}\right)$ & 0.0009 & 0.0117 & 0.0027 & 0.0119 & 0.0043 & 0.0133 & $0.4080(0.947)$ \\
\hline$\left(r_{2}, r_{3}\right)$ & 0.0006 & 0.0119 & 0.0015 & 0.0121 & 0.0036 & 0.0137 & $0.4076(0.950)$ \\
\hline \multicolumn{8}{|c|}{$\lambda=2.5$} \\
\hline \multirow[t]{2}{*}{ C.S } & \multicolumn{2}{|c|}{ MLE } & \multicolumn{2}{|c|}{ Lindley } & \multicolumn{2}{|c|}{ UMVUE } & \multirow[t]{2}{*}{ Lindley } \\
\hline & Bias & MSE & Bias & MSE & Bias & MSE & \\
\hline$\left(r_{1}, r_{1}\right)$ & 0.0009 & 0.0112 & 0.0024 & 0.0116 & 0.0026 & 0.0130 & $0.4054(0.949)$ \\
\hline$\left(r_{2}, r_{2}\right)$ & 0.0002 & 0.0119 & 0.0010 & 0.0120 & 0.0018 & 0.0123 & $0.4088(0.952)$ \\
\hline$\left(r_{3}, r_{3}\right)$ & 0.0009 & 0.0115 & 0.0017 & 0.0127 & 0.0067 & 0.0128 & $0.4117(0.946)$ \\
\hline$\left(r_{1}, r_{2}\right)$ & 0.0001 & 0.0118 & 0.0017 & 0.0119 & 0.0033 & 0.0131 & $0.4194(0.947)$ \\
\hline$\left(r_{1}, r_{3}\right)$ & 0.0016 & 0.0109 & 0.0042 & 0.0110 & 0.0048 & 0.0126 & $0.4096(0.946)$ \\
\hline$\left(r_{2}, r_{3}\right)$ & 0.0016 & 0.0119 & 0.0040 & 0.0121 & 0.0051 & 0.0132 & $0.4155(0.950)$ \\
\hline
\end{tabular}




\subsection{Data analysis}

Here we present a data analysis of the strength data reported by Badar and Priest [4]. This data, represent the strength measured in GPA for single carbon fibers, and impregnated 1000-carbon fiber tows. These data have been used previously by Raqab and Kundu [21], Kundu and Gupta [17] and Kundu and Raqab [18]. The data are presented in Table $\mathrm{V}$.

First, it was checked whether IERD can be used or not to analyze the two data sets, separately. The estimated parameters, for the first data set, are $\hat{\alpha}=74.6415, \hat{\lambda}=27.6710$ and for the second data set are $\hat{\beta}=16.6928, \hat{\lambda}=27.7890$. The Kolmogorov-Smirnov distances between the empirical distribution functions and the fitted distribution functions are 0.0826 and 0.0944 and corresponding p-values are 0.8623 and 0.6590 , respectively. Also, for the data set 1 and 2, the PP-plots are given in Figure 2. Based on the p-values, one can not reject the hypothesis that the data are coming from the above distribution.
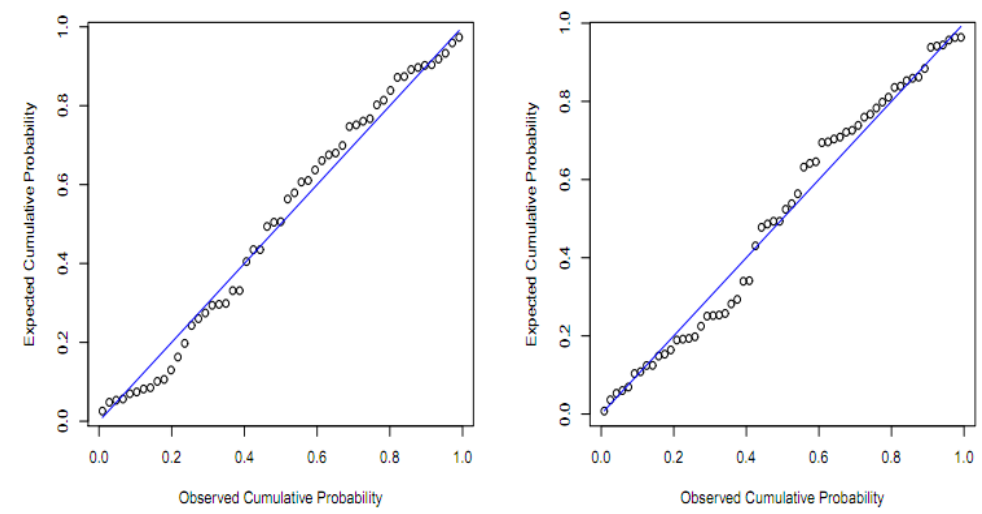

Fig. 2. The PP-plots for the first (left) and second (right) data set

Based on the complete data set, the proposed iterative procedure is used to compute the MLE. We plot the profile log-likelihood function of $\lambda$ in Figure 3. Since it is an unimodal function, it has the unique maximum. So, we start the iterative procedure with the initial values of $\lambda$ at such maximum 27.730. By this initial value, MLEs of $\lambda, \alpha$ and $\beta$ are $(27.731,75.2858,16.6029)$ and the MLE of $R$ is 0.8193 . Also, the corresponding $95 \%$ confidence interval become $(0.7556,0.8829)$. The Bayes estimate 
of $R$ with respect to improper priors is 0.8170 and the associated $95 \%$ credible interval is $(0.7597,0.8691)$.

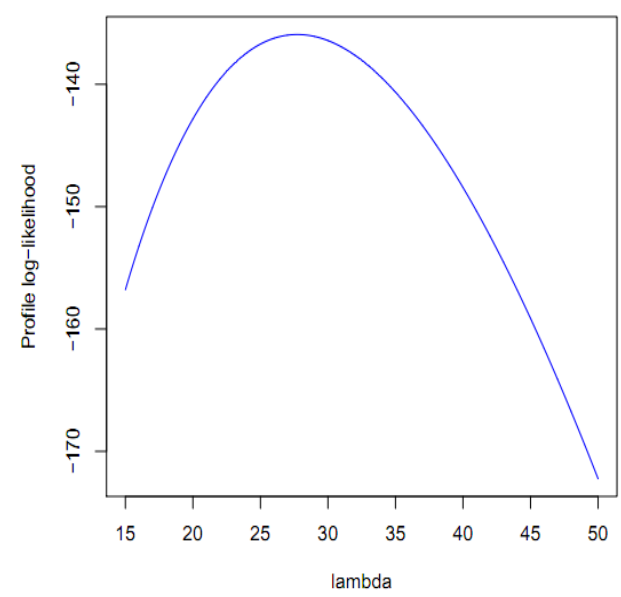

Fig. 3. The profile log-likelihood function of $\lambda$ for the real data

For illustrative purposes, two different progressively censored samples have been generated from the above data sets. The generated data and corresponding censored schemes have been presented in Table V. Based on Scheme 1, the MLE (by using the profile log-likelihood function) and Bayes estimates are 0.8972 and 0.8737 , respectively. The associated $95 \%$ asymptotic confidence interval and the credible interval are $(0.8001,0.9714)$ and $(0.7984,0.9593)$, respectively. Similarly, based on Scheme 2, the MLE and Bayes estimates are 0.9098 and 0.8968 , respectively. The associated $95 \%$ asymptotic confidence interval and the credible interval are $(0.7776,0.9697)$ and $(0.8033,0.9903)$, respectively. Clearly, the estimates obtained using Scheme 1, are closer to the estimates obtained by complete sample. 
Table V. Real Data.

\begin{tabular}{|c|c|c|c|c|c|c|c|c|c|c|}
\hline \multicolumn{11}{|c|}{ Real data set } \\
\hline \multicolumn{11}{|c|}{ Data Set $1(X)$} \\
\hline 1.865 & 1.944 & 1.958 & 1.966 & 1.997 & 2.006 & 2.021 & 2.027 & 2.055 & 2.063 & 2.098 \\
\hline 2.140 & 2.179 & 2.224 & 2.240 & 2.253 & 2.270 & 2.272 & 2.274 & 2.301 & 2.301 & 2.359 \\
\hline 2.382 & 2.382 & 2.426 & 2.434 & 2.435 & 2.478 & 2.490 & 2.511 & 2.514 & 2.535 & 2.554 \\
\hline 2.566 & 2.570 & 2.586 & 2.629 & 2.633 & 2.642 & 2.648 & 2.684 & 2.697 & 2.726 & 2.770 \\
\hline 2.773 & 2.800 & 2.809 & 2.818 & 2.821 & 2.848 & 2.880 & 2.954 & 3.012 & & \\
\hline \multicolumn{11}{|c|}{ Data Set $2(Y)$} \\
\hline 1.901 & 2.132 & 2.203 & 2.228 & 2.257 & 2.350 & 2.361 & 2.396 & 2.397 & 2.445 & 2.454 \\
\hline 2.474 & 2.518 & 2.522 & 2.525 & 2.532 & 2.575 & 2.614 & 2.616 & 2.618 & 2.624 & 2.659 \\
\hline 2.675 & 2.738 & 2.740 & 2.856 & 2.917 & 2.928 & 2.937 & 2.937 & 2.977 & 2.996 & 3.030 \\
\hline 3.125 & 3.139 & 3.145 & 3.220 & 3.223 & 3.235 & 3.243 & 3.264 & 3.272 & 3.294 & 3.332 \\
\hline 3.346 & 3.377 & 3.408 & 3.435 & 3.493 & 3.501 & 3.537 & 3.554 & 3.562 & 3.628 & 3.852 \\
\hline 3.871 & 3.886 & 3.971 & 4.024 & 4.027 & & & & & & \\
\hline \multicolumn{11}{|c|}{ Censored schemes and the corresponding data } \\
\hline \multicolumn{11}{|c|}{ Scheme 1: $R=[4,4,4,4,4,4,4,4,4,7], S=[4,4,4,4,4,4,4,4,4,14]$} \\
\hline$i, j$ & 1 & 2 & 3 & 4 & 5 & 6 & 7 & 8 & 9 & 10 \\
\hline$x_{i}$ & 1.966 & 2.055 & 2.224 & 2.274 & 2.382 & 2.490 & 2.566 & 2.642 & 2.770 & 2.821 \\
\hline$y_{j}$ & 2.454 & 2.532 & 2.624 & 2.856 & 2.977 & 3.145 & 3.264 & 3.377 & 3.537 & 3.871 \\
\hline \multicolumn{11}{|c|}{ Scheme 2: $R=[19,0,5,0,6,0,16], S=[19,0,5,0,6,0,23]$} \\
\hline$i, j$ & 1 & 2 & 3 & 4 & 5 & 6 & 7 & & & \\
\hline$x_{i}$ & 1.966 & 2.021 & 2.027 & 2.098 & 2.880 & 2.954 & 3.012 & & & \\
\hline$y_{j}$ & 3.628 & 3.852 & 3.871 & 3.886 & 3.971 & 4.024 & 4.027 & & & \\
\hline
\end{tabular}

\section{CONCLUSIONS}

In this paper, the estimation of the stress-strength parameter for IERD under progressive Type-II censoring has been considered. When the scale parameter is unknown different methods for estimating $R=P(X<Y)$ are used. It is observed that the MLE of $R$ can not be obtained in the closed form, therefore, an iterative procedure is applied to compute it. Also, we obtained the exact confidence interval of $R$. Moreover, we use the observed Fisher information matrix to obtain the asymptotic confidence interval. It is observed that even when the sample size is quite small the asymptotic confidence intervals work quite well. Also, two bootstrap confidence intervals were proposed that their performance is quite satisfactory. The Bayes estimate of $R$ and the corresponding credible interval can be obtained using the Gibbs sampling technique. It is observed that the MLE compares very well with the Bayes estimator in terms of biases and MSEs. Moreover, when the scale parameter is known, MLE, UMVUE and different Bayes estimators are computed. We observe that MLE provides the smallest biases and MSEs and the UMVUEs are the best 
second estimators. Also, the Lindley approximation behaves quite differently from the other. Monte Carlo simulations and data analysis are performed to check the performances of the different estimators.

This work has the potential to be applied in the context of reliability theory and censored data analysis. Further researches can be done in this direction by extending the progressive censored to the progressive hybrid and adaptive progressive hybrid censored IERD.

\section{REFERENCES}

Abouammoh, A.M. and Alshingiti, A.M. (2009). Reliability estimation of generalized inverted exponential distribution. Journal of Statistical Computation and Simulation. 79, 1301-1315.

Ahmad, K.E., Fakhry, M.E. and Jaheen, Z.F. (1997). Empirical Bayes estimation of $P(Y<X)$ and characterization of Burr-Type X model. Journal of Statistical Planning and Inference. 64, 297-308.

Asgharzadeh, A., Valiollahi, R. and Raqab, M.Z. (2011). Stress-strength reliability of Weibull distribution based on progressively censored samples. SORT. 35, 103-124.

Badar, M.G. and Priest, A.M. (1982). Statistical aspects of fiber and bundle strength in hybrid composites. In: Hayashi, T., Kawata, K., Umekawa, S. (Eds.). Progress in Science and Engineering Composites. ICCM-IV, Tokyo. 1129-1136.

Balakrishnan, N. and Aggarwala, R. (2000). Progressive censoring: theory, methods and applications. Birkhauser, Boston.

Birnbaum, Z.W. (1956). On a use of Mann-Whitney statistics. Proceedings of the third Berkley Symposium in Mathematics, Statistics and Probability. 1, 13-17.

Cao, J.H. and Cheng, K. (2006). An introduction to the reliability mathmematics. Beijing: Higher Education Press.

Chen, M.H. and Shao, Q.M. (1999). Monte Carlo estimation of Bayesian Credible and HPD intervals. Journal of Computational and Graphical Statistics. 8, 69-92.

Efron, B. (1982). The jackknife, the bootstrap and other re-sampling plans. Philadelphia, PA: SIAM, CBMSNSF Regional Conference Series in Applied Mathematics. 34.

Gradshteyn, I.S. and Ryzhik, I. M. (1994). Table of Integrals, Series, and Products. 5th ed., Academic Press, Boston, MA

Ghitany, M.E., Tuan, V.K. and Balakrishnan, N. (2012). Likelihood estimation for a general class of inverse exponentiated distributions based on complete and progressively censored data. Journal of Statistical Computation and Simulation. 84, 96-106.

Hall, P. (1988). Theoretical comparison of bootstrap confidence intervals. Annals of Statistics. 16, $927-$ 953. 
Johnson, N.L., Kotz, S. and Balakrishnan, N. (1994). Continuous Univariate Distributions. 2nd ed., Wiley, NewYork.

Kotz, S., Lumelskii, Y. and Pensky, M. (2003). The stress-strength model and its generalization: theory and applications. World Scientific, Singapore.

Krishnamoorthy, K., Mukherjee, S. and Guo, H. (2007). Inference on reliability in two-parameter exponential stress-strength model. Metrika. 65, 261-273.

Kundu, D. and Gupta, R.D. (2005). Estimation of $R=P(Y<X)$ for the generalized exponential distribution. Metrika. 61, 291-308.

Kundu, D. and Gupta, R.D. (2006). Estimation of $R=P(Y<X)$ for Weibull distribution. IEEE Transactions on Reliability. 55, 270-280.

Kundu, D. and Raqab, M.Z. (2009). Estimation of $R=P(Y<X)$ for three parameter Weibull distribution. Statistics and Probability Letters. 79, 1839-1846.

Lindley, D.V. (1980). Approximate Bayesian methods. Trabajos de Estadistica. 3, 281-288.

Lio, Y.L. and Tsai, T.R. (2012). Estimation of $\delta=P(X<Y)$ for Burr XII distribution based on the progressively first failure-censored samples. Journal of Applied Statistics. 39, 309-322.

Raqab, M.Z. and Kundu, D. (2005). Comparison of different estimators of $P(Y<X)$ for a scaled Burr Type X distribution. Communications in Statistics - Simulation and Computation. 34, 465-483.

Raqab, M.Z., Madi, M.T. and Kundu, D. (2008). Estimation of $R=P(Y<X)$ for the 3-parameter generalized exponential distribution. Communications in Statistics - Theory and Methods. 37, 2854 2864.

Rastogi, M.K. and Tripathi, Y.M. (2014). Estimation for an inverted exponentiated Rayleigh distribution under type II progressive censoring. Journal of Applied Statistics. 41, 2375-2405.

Saracoglu, B., Kinaci, I., Kundu, D. (2012). On estimation of $R=P(Y<X)$ for exponential distribution under progressive type-II censoring. Journal of Statistical Computation and Simulation. 82, 729-744.

Shoaee, S., and Khorram, E. (2015). Stress-strength reliability of a two-parameter bathtub-shaped lifetime distribution based on progressively censored samples. Communications in Statistics - Theory and Method. 44, 5306-5328.

Akram Kohansal

Department of Statistics, Imam Khomeini International University, Qazvin, Iran email: ak_kohansal@aut.ac.ir 